\title{
ON THE GALOIS STRUCTURE OF EQUIVARIANT LINE BUNDLES ON CURVES
}

\author{
By A. Agboola and D. Burns
}

\begin{abstract}
Let $k$ be a finite field, and let $X$ be a smooth, projective curve over $k$ with structure sheaf $\mathcal{O}$. Let $G$ be a finite group, and write $\mathrm{Cl}(\mathcal{O}[\mathrm{G}])$ for the reduced Grothendieck group of the category of $\mathcal{O}[G]$-vector bundles. In this paper we describe explicitly the subgroup of $\mathrm{Cl}(\mathcal{O}[G])$ which is generated by the classes arising from $G$-stable invertible sheaves on tame Galois covers of $X$ which have Galois group $G$.
\end{abstract}

Introduction. Let $k$ be a finite field of characteristic $p$, and let $G$ be a finite abelian group. Suppose that $f: Y \Leftrightarrow X$ is a tamely ramified Galois covering of smooth projective curves over $k$, with Galois group $G$. (We shall refer to such coverings as "tame $G$-covers" of $X$ ). In this paper we study the structure of $G$-stable line bundles on such curves $Y$.

In order to be more precise we let $\mathrm{Cl}\left(\mathcal{O}_{X}[G]\right)$, respectively $\mathrm{Cl}(k[G])$, denote the reduced Grothendieck group of $\mathcal{O}_{X}[G]$-vector bundles, respectively of finitely generated $k[G]$-modules which are cohomologically trivial for $G$. Suppose that $\mathcal{A}$ is a $G$-stable line bundle on $Y$. Then $f_{*} \mathcal{A}$ is an $\mathcal{O}_{X}[G]$-vector bundle, and it gives rise to a class $\left(f_{*} \mathcal{A}\right) \in \mathrm{Cl}\left(\mathcal{O}_{X}[G]\right)$. We shall say that an element of $\mathrm{Cl}\left(\mathcal{O}_{X}[G]\right)$ is realizable if it may be obtained in this manner for some choice of $Y$ and $\mathcal{A}$.

We shall combine techniques of $[\mathrm{C}],[\mathrm{M}]$ and $[\mathrm{Bu}]$ to obtain an explicit description of the subgroup $\Re_{X}(G)$ of $\mathrm{Cl}\left(\mathcal{O}_{X}[G]\right)$ which is generated by the subset of realizable classes. In conjunction with a Riemann-Roch theorem, these results are sufficient to describe explicitly those elements of $\mathrm{Cl}(k[G])$ which arise as the image of the vector bundles $f_{*} \mathcal{A}$ under the refined Euler-Poincaré characteristic map introduced by Chinburg in [Ch1], [Ch2] (see also [Ch,E]). Such a result is of interest in the context of "relative Galois module structure in a geometric setting" as indicated by Chinburg on p. 446 of [Ch2].

It is at the outset clear that there are differences between the case we consider here and the analogous number field case. For example, if $k^{c}$ is an algebraic closure of $k$ then any connected, finite, étale Galois cover of $X \times_{k} k^{c}$ has a group which is a quotient of the topological fundamental group of a Riemann surface of genus equal to the genus of $X$ (cf. [SGA1], exp. $X$ ). Since any tame cover of $X$ of $p$-power degree is necessarily étale it follows that not all abelian $p$-groups

Manuscript received May 17, 1996; revised July 22, 1997.

Research of the first author supported in part by an NSF postdoctoral research fellowship.

American Journal of Mathematics 120 (1998), 1121-1163. 
can occur as Galois groups of tame covers of $X$. By contrast, one knows that any finite abelian group can be realized as the Galois group of a tamely ramified extension of number fields. Perhaps more strikingly, we shall see that, in contrast to all "realizability results" for number fields (cf. for example [M], [By], [Mo]), the set of elements of $\mathrm{Cl}\left(\mathcal{O}_{X}[G]\right)$ which arise from the structure sheaves of tame Galois $G$-covers $Y \Leftrightarrow X$ do not in general form a subgroup. (It is however true that the subset of elements realized by structure sheaves of étale $G$-covers of $X$ is a subgroup of $\mathrm{Cl}\left(\mathcal{O}_{X}[G]\right)$.)

An outline of the contents of this paper is as follows. In $\S 1$, we describe various preliminary results concerning $\mathrm{Cl}\left(\mathcal{O}_{X}[G]\right)$. For brevity we shall only discuss the special cases which are relevant to the arguments of this paper (that is, either $p \nmid \# G$ or $G$ is cyclic of $p$-power order). A more systematic treatment of Grothendieck groups of vector bundles on certain types of ringed spaces over finite fields can be found in $[\mathrm{A}, \mathrm{Bu}]$.

Let $\mathfrak{R}_{X}^{0}(G)$ denote the subset of $\operatorname{Pic}\left(\mathcal{O}_{X}[G]\right)$ which is given by the classes arising from structure sheaves of tame $G$-covers of $X$. In $\S 2$ we give an explicit description of the subsets $\mathfrak{R}_{X}^{0}(G)$ and $\mathfrak{R}_{X}(G)$ in the case of étale covers of $p$ power degree (see Theorem 2.5) and that of tame covers of degree prime to $p$ (see Theorem 2.9). These results are then used in $\S 3$ to describe the image in $\mathrm{Cl}(k[G])$ of the refined Euler characteristic map of Chinburg.

In $\S 4$ we explain briefly how realizable classes in characteristic $p$ also give rise to classes in Grothendieck groups of categories of modules over rings of characteristic 0. Such classes arise naturally in connection with certain AdamsRiemann-Roch type theorems (cf. [Bu,Ch], [Ch,E,P,T]).

In $\S 5$, we describe how the results of $[\mathrm{M}]$ may be used to prove Theorem 2.9 (i) and (ii). Finally, in $\S 6$, we give a proof of Theorem 2.9 (iii) and (iv), using arguments along the lines of those contained in $[\mathrm{Bu}]$.

Acknowledgments. The authors are very grateful to both T. Chinburg and H. W. Lenstra for illuminating discussions and correspondence concerning this work.

1. Reduced Grothendieck groups of vector bundles. In this section we record some basic properties of the Grothendieck groups we shall use to classify the structure of vector bundles. For brevity, we shall only deal with the special cases which are needed for the results of this paper. A more systematic treatment of Grothendieck groups of bundles on certain types of ringed spaces over finite fields can be found in $[\mathrm{A}, \mathrm{Bu}]$.

We shall throughout write $\mathcal{O}_{X}$ for the structure sheaf of $X, K_{X}$ for the function field of $X$, and $k_{X}$ for the field of constants of $X$. When there is no danger of confusion these will be abbreviated to $\mathcal{O}, K$, and $k$ respectively.

We first give some general remarks on the classifying groups to be used here. To do this we let $\mathcal{O}[G]$ denote the ringed space on $X$ given by $U \mapsto$ 
$\mathcal{O}(U)[G]$ for each open subset $U$ of $X$. We let $\mathcal{V}(\mathcal{O}[G])$ denote the category of vector bundles (that is, locally-free modules) over $\mathcal{O}[G]$, and we write $K_{0}(\mathcal{O}[G]$ ) and $\mathrm{Cl}(\mathcal{O}[G])$ for the Grothendieck group and reduced Grothendieck group of $\mathcal{V}(\mathcal{O}[G])$ respectively.

Note that if $U$ is any nonempty affine open subset of $X$ then the section functor $\Gamma(U, \Leftrightarrow)$ is exact on $\mathcal{V}(\mathcal{O}[G])$ and so induces a homomorphism from $K_{0}(\mathcal{O}[G])$, respectively $\mathrm{Cl}(\mathcal{O}[G])$, to the Grothendieck group $K_{0}(\mathcal{O}(U)[G])$, respectively reduced Grothendieck group $\mathrm{Cl}(\mathcal{O}(U)[G])$, of the category of locally-free $\mathcal{O}(U)[G]$ modules. In this way the class in $K_{0}(\mathcal{O}[G])$, respectively $\mathrm{Cl}(\mathcal{O}[G])$, of a bundle $\mathcal{L}$ uniquely determines the class in $K_{0}(\mathcal{O}(U)[G])$, respectively $\mathrm{Cl}(\mathcal{O}(U)[G])$, of the space of sections $\mathcal{L}(U)$ for each nonempty affine open subset $U$ of $X$. However, not even the stable $k[G]$-isomorphism class of the global sections of a bundle is in general determined by its class in $K_{0}(\mathcal{O}[G])$. Moreover, if $\mathcal{V}$ is a bundle, then knowledge of the class of $\mathcal{V}(U)$ in $K_{0}(\mathcal{O}(U)[G])$ for each open affine subset $U$ of $X$ is in general not sufficient even to determine the class of $\mathcal{V}$ in $\mathrm{Cl}(\mathcal{O}[G])$.

For any ringed space $\mathcal{A}$ we write $\operatorname{det}_{\mathcal{A}}$ for the determinant (that is, top exterior power) functor on the category of $\mathcal{A}$-vector bundles.

Proposition 1.1. The functor $\operatorname{det}_{\mathcal{O}[G]}$ induces an isomorphism between $\mathrm{Cl}(\mathcal{O}[G])$ and $\operatorname{Pic}(\mathcal{O}[G])$.

Proof. We let $f_{\mathcal{O}[G]}: X(\mathcal{O}[G]) \rightarrow X$ denote the affine spectrum of $\mathcal{O}[G]$ (cf. [EGA1], Chapter $1, \S 9)$. For each natural number $n$ the direct image functor $f_{\mathcal{O}[G] \text {,* }}$ is exact from the category of bundles on $X(\mathcal{O}[G])$ which are of constant rank $n$ to the category of $\mathcal{O}[G]$-bundles on $X$ which are of rank $n$. This functor has an (exact) two-sided inverse and so induces isomorphisms $\mathrm{Cl}(X(\mathcal{O}[G])) \stackrel{\sim}{\rightarrow} \mathrm{Cl}(\mathcal{O}[G])$ and $\operatorname{Pic}(X(\mathcal{O}[G])) \stackrel{\sim}{\rightarrow} \operatorname{Pic}(\mathcal{O}[G])$. Taken together these isomorphisms reduce the proof of Proposition 1.1 to showing that $\operatorname{det}_{\mathcal{O}_{X(\mathcal{O}[G])}}$ induces an isomorphism between $\mathrm{Cl}(X(\mathcal{O}[G]))$ and $\mathrm{Pic}(X(\mathcal{O}[G]))$. Moreover, since each connected component of $X(\mathcal{O}[G])$ is a noetherian curve which possesses ample line bundles, this follows directly form ([F,L], Chapter V, Corollary 3.10 and appendix, and Chapter III, Theorem 1.7).

COROllary 1.2. If $\mathcal{L}$ is an invertible $\mathcal{O}[G]$-module then the following conditions are equivalent:

(i) $\mathcal{L}$ has trivial class in $\mathrm{Cl}(\mathcal{O}[G])$.

(ii) $\mathcal{L}$ is a free $\mathcal{O}[G]-$ module.

(iii) $\mathcal{L}$ is a stably free $\mathcal{O}[G]$-module.

Proof. Since $\operatorname{det}_{\mathcal{O}[G]} \mathcal{L} \cong \mathcal{L}$ the implication from (i) to (ii) is a consequence of Proposition 1.1, whilst those from (ii) to (iii) and (iii) to (i) are obvious.

For the purposes of this paper it will suffice for us to have explicit descriptions of Pic $(\mathcal{O}[G])$ for the cases that $G$ is a cyclic $p$-group or has order coprime to $p$, and so we shall only discuss these special cases. 
To deal with the case that $G$ is cyclic of $p$-power order we use the Witt vector cohomology groups of Serre. For each positive integer $i$ we thus let $\mathcal{W}_{i}$ denote the sheaf of Witt vectors of length $i$ on $X$ as introduced in [S1]. If $G$ has order $p^{N}$ then for each positive integer $i$ we let $r_{i}$ denote the least positive integer such that $p^{r_{i}} \geq p^{N} / i$.

Let $T$ be an indeterminate. For each $k$-algebra $A$ the group $A[G]^{*}$ can be identified with the group of units of the $\operatorname{ring} R(A):=A[T] /\left(T^{p^{N}}\right)$. We fix such an identification by choosing a generator $g$ of $G$ and letting the class $t$ of $T$ in $R(A)$ correspond to the element $g \Leftrightarrow 1 \in A[G]$. We write $E(\Leftrightarrow)$ for the Artin-Hasse exponential function on Witt vectors, and recall that each element $\alpha$ of $R(A)^{*}$ can be written uniquely as a product

$$
\alpha=\alpha_{0} \times \prod_{\substack{1 \leq i \leq p^{N} \\(i, p)=1}} E\left(\alpha_{i} t^{i}\right),
$$

where here $\alpha_{0} \in A^{*}$, and each $\alpha_{i} \in W_{r_{i}}(A)$ ([S2], Chapitre V, Proposition 9). By applying this decomposition with $A=\mathcal{O}(U)$ for each open subset $U$ of $X$ one obtains an isomorphism of sheaves of groups on $X$

$$
\mathcal{O}[G]^{*} \stackrel{\sim}{\rightarrow} \mathcal{O}^{*} \times \prod_{\substack{1 \leq i \leq p^{N} \\(i, p)=1}} \mathcal{W}_{r_{i}}
$$

We let $W(\mathcal{O}[G])$ denote the direct sum

$$
\operatorname{Pic}(\mathcal{O}) \oplus \bigoplus_{\substack{1 \leq i \leq p^{N} \\(i, p)=1}} H^{1}\left(X_{\mathrm{Zar}}, \mathcal{W}_{r_{i}}\right)
$$

In conjunction with the canonical identification $\operatorname{Pic}(\mathcal{O}[G]) \cong H^{1}\left(X_{\mathrm{Zar}}, \mathcal{O}[G]^{*}\right)$ one obtains from (1.1) an isomorphism (dependent upon the choice of generating element $g$ )

$$
\theta_{g}: \operatorname{Pic}(\mathcal{O}[G]) \stackrel{\sim}{\rightarrow} W(\mathcal{O}[G])
$$

We now turn to consider the case that $p \nmid \# G$. In order to describe realizable classes in this case it is convenient to first reinterpret Pic $(\mathcal{O}[G])$ in terms of the class group introduced by Chapman in [C]. To do this we fix an algebraic closure $k^{c}$ of $k$ and a separable algebraic closure $K^{c}$ of $K$ which contains $k^{c}$. We let $\Omega_{k}$ and $\Omega_{K}$ denote the Galois groups of $k^{c} / k$ and $K^{c} / K$ respectively. We let $K^{\prime}$ denote the field $K \otimes_{k} k^{c}$ which is a Galois extension of $K$ (in $K^{c}$ ) of group $\Omega_{k}$. For each group $G$ we set $\hat{G}=\operatorname{Hom}\left(G,\left(k^{c}\right)^{*}\right)$, and we let $R_{G}$ denote the ring of $k^{c}$-valued characters of $G$. For each group $G$ which has order coprime to $p$ the Chapman class group $\mathrm{Ch} \mathrm{Cl}(\mathcal{O}[G])$ is as an abstract group equal to $\operatorname{Hom}_{\Omega_{K}}\left(R_{G}, \operatorname{Pic}\left(K^{\prime}\right)\right)$, and is such that each $\mathcal{O}[G]$-bundle $\mathcal{V}$ gives a natural class $[\mathcal{V}] \in \mathrm{ChCl}(\mathcal{O}[G])$. 
We shall only need to describe $[\mathcal{V}]$ explicitly for the case of a line bundle $\mathcal{V}$. In this case the stalk $\mathcal{V}_{x}$ is freely generated over $\mathcal{O}_{X}[G]$ by an element $\mathrm{v}_{x}$, say, for each $x \in X$. We write $X_{0}$ for the set of closed points of $X$. Since each point $x \in X_{0}$ is a specialization of the unique generic point $\eta$ of $X$ there is a natural map from each $\mathcal{V}_{x}$ to the generic stalk $V_{\eta}$. In this way each stalk $\mathcal{V}_{x}$ can be regarded as an $\mathcal{O}_{x}[G]$-lattice in the free $K[G]$-module $\mathcal{V}_{\eta}$, so that $\mathrm{v}_{x}=\lambda_{x} \cdot \mathrm{v}_{\eta}$ for a unique $\lambda_{x} \in K[G]$. For each integer $s$ we let $M_{s}\left(K^{\prime}\right)$ denote the ring of $s \times s$ matrices with coefficients in $K^{\prime}$, and let $G L_{s}\left(K^{\prime}\right)$ denote the unit group of $M_{s}\left(K^{\prime}\right)$. We extend each representation $\chi: G \Leftrightarrow G L_{s}\left(K^{\prime}\right)$ of $G$ to a $K^{\prime}$-algebra homomorphism $\tilde{\chi}: K^{\prime}[G] \Leftrightarrow M_{s}\left(K^{\prime}\right)$, and define

$$
D(\mathcal{V})_{\chi}:=\sum_{w} \operatorname{Val}_{w}\left(\operatorname{det}\left(\tilde{\chi}\left(\lambda_{x(w)}\right)\right) w\right.
$$

where here $w$ runs through the places of $K^{\prime}, \operatorname{Val}_{w}(\Leftrightarrow)$ denotes the valuation of $K^{\prime}$ corresponding to $w$, and $x(w) \in X$ corresponds to the prime divisor of $K$ which lies beneath $w$. It is not difficult to see that each $D(\mathcal{V})_{\chi}$ belongs to $\operatorname{Div}\left(K^{\prime}\right)$. Furthermore, writing $\left[D\left(\mathcal{V}_{\chi}\right]\right.$ for the image of $D(\mathcal{V})_{\chi}$ in $\operatorname{Pic}\left(K^{\prime}\right),\left[D(\mathcal{V})_{\chi}\right]$ is independent of the choice of each $\lambda_{x(w)}$ and is such that the map $[\mathcal{V}] \in \operatorname{Hom}\left(R_{G}, \operatorname{Pic}\left(K^{\prime}\right)\right)$ defined by sending each character $\chi$ to the class $\left[D(\mathcal{V})_{\chi}\right]$ actually belongs to $\operatorname{Hom}_{\Omega_{K}}\left(R_{G}\right.$, Pic $\left.\left(K^{\prime}\right)\right)$ (cf. [C], Lemmas 1 and 2 and Proposition 1).

Proposition 1.3. (Chapman, [C]) Let $G$ be a finite abelian group which has order coprime to $p$. Then the map

$$
\theta_{\mathcal{O}[G]}: \operatorname{Pic}(\mathcal{O}[G]) \stackrel{\sim}{\rightarrow} \operatorname{Hom}_{\Omega_{K}}\left(R_{G}, \operatorname{Pic}\left(K^{\prime}\right)\right)
$$

which is induced by sending each line bundle $\mathcal{L}$ to the element $[\mathcal{L}]$ defined above is an isomorphism.

Proof. This result is not stated explicitly in [C] and so we shall quickly sketch a proof. For each $K$-algebra $A$ we let $J_{K}(A)$ denote the associated group of ideles.

For each $\mathcal{O}[G]$-line bundle $\mathcal{L}$ we choose an element $\left(\ell_{x}\right)_{x \in X_{0}} \in \prod_{x \in X_{0}} K[G]^{*}$ as above. The $\left(\ell_{x}\right)_{x \in X_{0}} \in J_{K}(K[G])$, and the association $\mathcal{L} \mapsto\left(\lambda_{x}\right)_{x \in X_{0}}$ induces a well-defined group isomorphism

$$
\operatorname{Pic}(\mathcal{O}[G]) \cong \frac{J_{K}(K[G])}{(K[G])^{*} \prod_{x \in X_{0}}\left(\mathcal{O}_{x}[G]\right)^{*}}
$$

Let $B$ be a $k$-algebra. Since $p \nmid \# G$ we may identify $\left(B \otimes_{k} k^{c}\right)[G]$ with the $B \otimes_{k} k^{c}$-algebra of functions from $\hat{G}$ to $B \otimes_{k} k^{c}$ with pointwise operations; so we have $\left(B \otimes_{k} k^{c}\right)[G]=\operatorname{Map}\left(\hat{G}, B \otimes_{k} k^{c}\right)$ where, if $\beta \in\left(B \otimes_{k} k^{c}\right)[G]$ and $\chi \in \hat{G}$ then $\beta(\chi):=\chi(\beta)$ for each $\chi \in \hat{G}$. The group of units $\left(B \otimes_{k} k^{c}\right)[G]^{*}$ may thus be identified with $\operatorname{Hom}\left(\mathbb{Z}[\hat{G}],\left(B \otimes_{k} k^{c}\right)^{*}\right)$, and so by taking fixed points under the action of $\Omega_{K}$ one obtains an identification $B[G]^{*} \cong \operatorname{Hom}_{\Omega_{K}}\left(\mathbb{Z}[\hat{G}],\left(B \otimes_{k} k^{c}\right)^{*}\right)$. 
By applying this isomorphism with $B$ equal to $K$ and $\mathcal{O}_{x}$ for each $x \in X_{0}$ one obtains an isomorphism

$$
\frac{J_{k}(K[G])}{(K[G])^{*} \prod_{x \in X_{0}}\left(\mathcal{O}_{x}[G]\right)^{*}} \cong \frac{\operatorname{Hom}_{\Omega_{K}}\left(R_{G}, \prod_{x \in X_{0}}\left(K_{x}^{\prime}\right)^{*}\right)}{\operatorname{Hom}_{\Omega_{K}}\left(R_{G},\left(K^{\prime}\right)^{*}\right) \operatorname{Hom}_{\Omega_{K}}\left(R_{G}, \prod_{x \in X_{0}}\left(\mathcal{O}_{x} \otimes_{k} k^{c}\right)^{*}\right)} .
$$

There is a natural morphism from this quotient to the group $\operatorname{Hom}_{\Omega_{K}}\left(R_{G}, \operatorname{Pic}\left(K^{\prime}\right)\right)$ and, by using the fact that $\operatorname{Pic}(X)$ has Galois descent as a functor on $\operatorname{Spec}(k)_{e t}$, one can show that this morphsim is bijective (cf. [C], Theorem 1).

This "Hom-description" of Pic $(\mathcal{O}[G])$ is well adapted to explicit computation of arithmetical classes (cf. $\S 5$ and $\S 6$ ). It is also amendable to studying functorial behavior under extension or restriction of scalars. In this direction it is convenient to record the following result:

LEMMA 1.4. Let $G$ be a finite abelian group which has order coprime to $p$. The for each subgroup $J$ of $G$ there is a natural morphism $\operatorname{ind}_{J}^{G}$ : $\operatorname{Pic}(\mathcal{O}[J]) \rightarrow$ $\operatorname{Pic}(\mathcal{O}[G])$ which is induced by the functor $\otimes_{\mathcal{O}[J]} \mathcal{O}[G]$. If we also let $\operatorname{ind}_{J}^{G}$ denote the homomorphism

$$
\operatorname{Hom}_{\Omega_{K}}\left(R_{J}, \operatorname{Pic}\left(K^{\prime}\right)\right) \rightarrow \operatorname{Hom}_{\Omega_{K}}\left(R_{G}, \operatorname{Pic}\left(K^{\prime}\right)\right)
$$

which is induced by the restriction map on characters $R_{G} \rightarrow R_{J}$, then the following diagram commutes

$$
\begin{aligned}
& \operatorname{Pic}(\mathcal{O}[J]) \stackrel{\theta_{\mathcal{O}[J]}}{\longrightarrow} \operatorname{Hom}_{\Omega_{K}}\left(R_{J}, \operatorname{Pic}\left(K^{\prime}\right)\right) \\
& \operatorname{ind}_{J}^{G} \downarrow \quad \downarrow^{\operatorname{ind}_{J}^{G}} \\
& \operatorname{Pic}(\mathcal{O}[G]) \stackrel{\theta_{\mathcal{O}[G]}}{\longrightarrow} \operatorname{Hom}_{\Omega_{K}}\left(R_{G}, \operatorname{Pic}\left(K^{\prime}\right)\right) \text {. }
\end{aligned}
$$

Proof. Just as for ([F], Theorem 12).

2. Realizable bundle classes. Throughout this section $G$ is a finite abelian group. We write $\Re_{X}^{0}(G)$ for the subset of $\operatorname{Pic}(\mathcal{O}[G])$ given by the classes of structure sheaves in all tame Galois $G$-covers of $X$, and we let $\mathfrak{R}_{X}(G)$ denote the subgroup of $\mathrm{Pic}(\mathcal{O}[G])$ which is generated by the classes of arbitrary $G$-stable invertible sheaves arising from tame Galois $G$-covers of $X$. Our aim in this section is to explicitly describe the subsets $\Re_{X}^{0}(G)$ and $\Re_{X}(G)$.

We let $P$, respectively $H$, denote the maximal subgroup of $G$ which has order a power of $p$, respectively order coprime to $p$. For any tame $G$-cover $f: Y \Leftrightarrow X$ we let $f^{p}: Y(p) \rightarrow X$, respectively $f^{\prime}: Y\left(p^{\prime}\right) \rightarrow X$, denote the subcover which corresponds by Galois theory to the subgroup $P$, respectively $H$, of $G$ (so that 
$K_{Y(p)}=K_{Y}^{P}$ and $\left.K_{Y\left(p^{\prime}\right)}=K_{Y}^{H}\right)$. Since $f$ is tame the morphism $f^{\prime}$ is necessarily étale. We first record an easy reduction step.

LeMma 2.1. For any $G$-stable invertible sheaf $\mathcal{A}$ on $Y$, the $\mathcal{O}[G]$-bundle $f_{*} \mathcal{A}$ is isomorphic to a bundle $f_{*}^{p} \mathcal{A}^{\prime} \otimes \mathcal{O} f_{*}^{\prime} \mathcal{O}_{Y\left(p^{\prime}\right)}$ for some $G / P$-stable invertible $\mathcal{O}_{Y(p)^{-}}$ sheaf $\mathcal{A}^{\prime}$ on $Y(p)$. Moreover, if $P$ is the direct product of cyclic groups $P_{i}, i=$ $1,2, \ldots, s$, and $f_{i}^{\prime}: Y\left(p^{\prime}\right)_{i} \rightarrow X$ is the subcover of $f^{\prime}$ corresponding to the subgroup $\prod_{1 \leq j \leq s, j \neq i} P_{j}$ of $P$, then the $\mathcal{O}[P]$-bundle $f_{*}^{\prime} \mathcal{O}_{Y\left(p^{\prime}\right)}$ is isomorphic to the tensor product $f_{1, *}^{\prime} \mathcal{O}_{Y\left(p^{\prime}\right)_{1}} \otimes_{\mathcal{O}} f_{2, *}^{\prime} \mathcal{O}_{Y\left(p^{\prime}\right)_{2}} \otimes_{\mathcal{O}} \cdots \otimes_{\mathcal{O}} f_{s, *}^{\prime} \mathcal{O}_{Y\left(p^{\prime}\right)_{s}}$.

Proof. Let $U$ be any affine open subset of $X$. The induced morphism $Y \rightarrow$ $Y(p)$ is étale and hence the $G$-stable ideal $f_{*} \mathcal{A}(U)$ of $f_{*} \mathcal{O}_{Y}(U)$ is of the form $\mathcal{A}^{\prime}(U) f_{*} \mathcal{O}_{Y}(U)$ for some $G / P$-stable ideal $\mathcal{A}^{\prime}(U)$ of $f_{*}^{p} \mathcal{O}_{Y(p)}(U)$. In addition, since $K_{Y(p)}$ and $K_{Y\left(p^{\prime}\right)}$ are linearly disjoint and of coprime ramification over $K$ there is a natural isomorphism $f_{*} \mathcal{O}_{Y}(U) \cong f_{*}^{p} \mathcal{O}_{\left.Y_{(}\right)}(U) \otimes \mathcal{O}_{(U)} f_{*}^{\prime} \mathcal{O}_{Y\left(p^{\prime}\right)}(U)$ (cf. the argument of ([L2], §II.3, Proposition 17)), and so one has

$$
f_{*} \mathcal{A}(U) \cong \mathcal{A}^{\prime}(U) \otimes \mathcal{O}_{(U)} f_{*}^{\prime} \mathcal{O}_{Y\left(p^{\prime}\right)}(U)
$$

It is clear that the $\mathcal{O}[G / P]$-sheaf $U \mapsto \mathcal{A}^{\prime}(U)$ is equal to $f_{*}^{p} \mathcal{A}^{\prime}$ for some $G / P$ stable invertible $\mathcal{O}_{Y(p)}$-sheaf $\mathcal{A}^{\prime}$ and that the isomorphisms (2.1) glue to give an isomorphism $f_{*} \mathcal{A} \cong f_{*}^{p} \mathcal{A}^{\prime} \otimes \mathcal{O} f_{*}^{\prime} \mathcal{O}_{Y\left(p^{\prime}\right)}$.

A similar argument will prove the second assertion.

This result implies that when one analyzes the contribution from the étale cover $f^{\prime}: Y\left(p^{\prime}\right) \rightarrow X$ to the realizable classes in $\mathrm{Cl}(\mathcal{O}[G])$ it is sufficient to consider only the structure sheaf $\mathcal{O}_{Y\left(p^{\prime}\right)}$.

We next note that the module structure of the space of global sections of the structure sheaf is always easy to determine.

Proposition 2.2. Letf: $Y \rightarrow X$ be a Galois $G$-cover of $X$. If $G_{0}$ is the subgroup of $G$ which corresponds by Galois theory to the maximal constant field extension of $K$ in $K_{Y}$ then $\mathcal{O}_{Y}(Y)$ is $k[G]$-isomorphic to $k\left[G / G_{0}\right]$. In particular, iff is tame then $f_{*} \mathcal{O}_{Y}$ has trivial class in $\mathrm{Cl}(\mathcal{O}[G])$ if and only if $K_{Y}$ is a constant field extension of $K$.

Proof. The maximal constant field extension of $K$ in $K_{Y}$ is $K k_{Y}$ with $k_{Y}$ equal to the constant field $\mathcal{O}_{Y}(Y)$ of $Y$. The first assertion thus follows by the normal basis theorem. If now $f$ arises by base changing $X$ through a (constant) field extension $k^{\prime}$ of $k$ then $f_{*} \mathcal{O}_{Y}(U)=\mathcal{O}(U) k^{\prime}$ for each affine open subset $U$ of $X$. One checks easily that any explicit isomorphism $k^{\prime} \cong k[G]$ extends to give an isomorphism of $\mathcal{O}[G]$-modules between $f_{*} \mathcal{O}_{Y}$ and $\mathcal{O}[G]$. Finally, we recall that if $f_{*} \mathcal{O}_{Y}$ has trivial class in $\mathrm{Cl}(\mathcal{O}[G])$ then it is a free $\mathcal{O}[G]$-module of rank 1 (cf. Corollary 1.2). By taking sections over $X$ it follows that $k_{Y}$ is isomorphic to $k[G]$ and hence that $K_{Y}$ must be a constant field extension of $K$. 
2.1. Étale covers of $p$-power degree. In this subsection we shall describe the subset of Pic $(\mathcal{O}[P])$ which arises from the classes of structure sheaves in étale Galois $P$-covers of $X$.

From Proposition 2.2 the module structure of the space of global sections of the structure sheaf of an étale $P$-cover is explicitly known. We note now that the $P$-structure of the restriction of the structure sheaf to each proper affine open subset of $X$ is also easily described.

LEMMA 2.3. For each nonempty affine open subset $U$ of $X$ the restriction of $f_{*}^{\prime} \mathcal{O}_{Y\left(p^{\prime}\right)}$ to $U$ is a free rank one $\left.\mathcal{O}\right|_{U}[P]$-module.

Proof. Here we set $\mathcal{O}^{\prime}=f_{*}^{\prime} \mathcal{O}_{Y\left(p^{\prime}\right)}$. By Noether's theorem (cf. the proof of $[\mathrm{F}]$, Chapter I, Theorem 3) one know that $\mathcal{O}^{\prime}$ is an invertible $\mathcal{O}[P]$-module, and that the "trace element" $\sum_{g \in P} g$ induces a surjective morphism of $\mathcal{O}[P]$-modules $\mathcal{O}^{\prime} \rightarrow \mathcal{O}$. For any nonempty affine open subset $U$ of $X$ we write $I(U, P)$ for the augmentation ideal of $\mathcal{O}(U)[P]$. Since $\mathcal{O}(U)[P]$ is $I(U, P)$-adically complete one has a natural isomorphism $K_{0}(\mathcal{O}(U)[P]) \stackrel{\sim}{\rightarrow} K_{0}(\mathcal{O}(U))$ (cf. [Ba], p. 449, Proposition 1.3(0)) and so $\mathcal{O}^{\prime}(U)=\mathcal{O}(U)[P] y$ for any element $y \in \mathcal{O}^{\prime}(U)$ for which $\sum_{g \in P} g y \in \mathcal{O}(U)^{*}$.

Rather than referring to $[\mathrm{Ba}]$ to obtain such a description of $\mathcal{O}^{\prime}(U)$ one can also proceed more directly in this case as follows: for each $x \in U$ there exists $y_{x} \in \mathcal{O}_{x}^{\prime}$ which freely generates the stalk $\mathcal{O}_{x}^{\prime}$ over $\mathcal{O}_{x}[P]$. Moreover, there exists $y \in \mathcal{O}^{\prime}(U)$ such that $\sum_{g \in P} g y \in \mathcal{O}(U)^{*}$. For each $x \in U$ we choose $\lambda_{x} \in \mathcal{O}_{x}[P]$ such that $y=\lambda_{x} y_{x}$. With $\epsilon: K[P] \rightarrow K$ denoting the augmentation map one has $\sum_{g \in P} g y=\epsilon\left(\lambda_{x}\right) \sum_{g \in P} g y_{x}$ so that in particular $\epsilon\left(\lambda_{x}\right) \in \mathcal{O}_{x}^{*}$. But $\mathcal{O}_{x}[P]$ is a local ring with maximal ideal given by those elements whose image under $\epsilon$ lies in the maximal ideal of $\mathcal{O}_{x}$. Thus one has $\lambda_{x} \in \mathcal{O}_{x}[P]^{*}$ so that $\mathcal{O}_{x}[P] y=\mathcal{O}_{x}[P] \lambda_{x} y_{x}=$ $\mathcal{O}_{x}[P] y_{x}=\mathcal{O}_{x}^{\prime}$ for each $x \in U$. Since clearly $\mathcal{O}\left(U^{\prime}\right)[P] y \subseteq \mathcal{O}^{\prime}\left(U^{\prime}\right)$ for each affine open subset $U^{\prime} \subset U$ it follows that $\mathcal{O}^{\prime}\left(U^{\prime}\right)$ is a free $\mathcal{O}\left(U^{\prime}\right)[P]$-module with basis $y$. Any such element $y$ thus induces an isomorphism of $\left.\mathcal{O}\right|_{U}[P]$-modules between $\left.\mathcal{O}^{\prime}\right|_{U}$ and $\left.\mathcal{O}\right|_{U}[P]$.

To proceed further we now specialize to the case that $P$ is a cyclic group of order $p^{N}$ (as indeed we may by Lemma 2.1). We first recall that in this case Artin-Schreier theory gives a description of the group $\pi^{1}(X, P)$ of isomorphism classes of $P$-torsors over $X$ (or equivalently, of étale Galois $P$-covers of $X$ ) in terms of Witt vector cohomology groups.

If $A$ is any abelian group with an endomorphism $\alpha$ then we shall write $A^{\alpha=1}$ and $A_{\alpha-1}$ for the subgroup $\{a \in A: \alpha a=a\}$ and the quotient of $A$ by $\{\alpha a \Leftrightarrow a: a \in A\}$ respectively.

Proposition 2.4. (Serre, [S1], Proposition 13; [Mi], Chapter 3, Proposition 4.12) Choose a generator $g$ of $P$, and so identify $P$ with $\mathbb{Z} / p^{N}$. Then there are natural homomorphisms $\delta_{g}$ and $\phi_{g}$ (dependent upon the choice of $g$ ) and $a$ 
short exact sequence of abelian groups

$$
0 \rightarrow W_{N}(k)_{F-1} \stackrel{\delta_{g}}{\Leftrightarrow} \pi^{1}(X, P) \stackrel{\phi_{g}}{\Leftrightarrow} H^{1}\left(X_{\mathrm{Zar}}, \mathcal{W}_{N}\right)^{F=1} \rightarrow 0
$$

(Here $F$ is the map induced by the Frobenius endomorphism $t \mapsto t^{p}$ of $k$.)

In this case one knows also that the map

$$
\psi_{P}: \pi^{1}(X, P) \rightarrow \operatorname{Pic}(\mathcal{O}[P])
$$

which is induced by sending a $P$-torsor $f: Y \rightarrow X$ to the class of the bundle $f_{*} \mathcal{O}_{Y}$ is a homomorphism (cf. [W], Theorem 5). The kernel of $\psi_{P}$ can be described in terms of symmetric Hochschild cohomology (cf. ibid., p. 182) but it is for our purposes more convenient to relate this kernel directly to the short exact sequence of Proposition 2.4. In the statement of the next result we shall use the notation of Proposition 2.4 .

THEOREM 2.5. The kernel of $\psi_{P}$ coincides with the image of $\delta_{g}$. In particular, the set $\Re_{X}^{0}(P)$ is a subgroup of $\mathrm{Pic}(\mathcal{O}[P])$ which is isomorphic to $H^{1}\left(X_{\mathrm{Zar}}, \mathcal{W}_{N}\right)^{F=1}$.

Noting that $\Re_{X}^{0}(P)=\operatorname{Image}\left(\psi_{P}\right)$ it is at once clear that if $\operatorname{ker}\left(\psi_{P}\right)=$ Image $\left(\delta_{g}\right)$ then the second assertion of Theorem 2.5 follows from Proposition 2.4. To prove Theorem 2.5 it therefore suffices to show that $\operatorname{ker}\left(\psi_{P}\right)=$ Image $\left(\delta_{g}\right)$, and to prove this equality we shall simply describe both sides explicitly.

Lemma 2.6. Let $f: Y \rightarrow X$ be an étale Galois $P$-cover of $X$. Then $\psi_{P}(Y)=0$ if and only if there exists a subgroup $Q$ of $P$ such that the generic fibre of $Y$ is the Galois $P$-algebra $\prod_{g \in P / Q} K_{Q}$ where here $K_{Q}$ is the constant field extension of $K$ of group $Q$ (with respect to some identification of $\mathrm{Gal}\left(K_{Q} / K\right)$ with $Q$ ).

Proof. The generic fibre $Y_{\eta}$ of $Y$ is a Galois $P$-algebra over $K$ so that there is a subgroup $Q$ of $P$, and an unramified Galois field extension $L$ of $K$ of group (isomorphic to) $Q$, such that $Y_{\eta} \cong \prod_{g \in P / Q} L$. If now $L$ corresponds to the étale $Q$-cover $f^{\prime}: Z \rightarrow X$ then $f_{*} \mathcal{O}_{Y} \cong \prod_{g \in P / Q} f_{*}^{\prime} \mathcal{O}_{Z}$ and so, upon taking sections over $X$, one obtains a $k[P]$-module isomorphism between $k_{Y}$ and $\operatorname{ind}_{Q}^{P} k_{Z}$. Now, as a consequence of Proposition 2.2, one knows that

$$
\psi_{P}(Y)=0 \Leftrightarrow k_{Y} \cong k[P] \Leftrightarrow k_{Z} \cong k[Q]
$$

and this last isomorphism is valid if and only if $Z$ is the $Q$-cover of $X$.

We must now be more precise concerning the map $\delta_{g}$ used in Proposition 2.4. We let $X_{0}, X_{1}, \ldots, X_{N-1}$ be $N$ independent indeterminates. For each vector $\alpha \in W_{N}(k)$ we let $\left(X_{0}, X_{1}, \ldots, X_{N-1} ; \alpha\right)$ denote the ideal of the polynomial ring $k\left[X_{0}, X_{1}, \ldots, X_{N-1}\right]$ which is generated by those relations between the 
indeterminates which are equivalent to the equality

$$
\begin{gathered}
F\left(X_{0}, X_{1}, \ldots, X_{N-1}\right) \Leftrightarrow\left(X_{0}, X_{1}, \ldots, X_{N-1}\right)=\alpha \\
\text { in } \quad W_{N}\left(k\left[X_{0}, X_{1}, \ldots, X_{N-1}\right]\right) .
\end{gathered}
$$

We define an action of $P$ on the $k$-algebra

$$
k(\alpha):=k\left[X_{0}, X_{1}, \ldots, X_{N-1}\right] /\left(X_{0}, X_{1}, \ldots, X_{N-1} ; \alpha\right)
$$

by specifying that the action of the chosen generator $g$ should be that induced by the automorphism of $W_{N}\left(k\left[X_{0}, X_{1}, \ldots, X_{N-1}\right]\right)$ given by

$$
\begin{gathered}
\left(X_{0}, X_{1}, \ldots, X_{N-1}\right) \mapsto\left(X_{0}, X_{1}, \ldots, X_{N-1}\right)+1 \\
\text { in } \quad W_{N}\left(k\left[X_{0}, X_{1}, \ldots, X_{N-1}\right]\right) .
\end{gathered}
$$

With respect to this action the natural morphism

$$
X_{g}(\alpha):=\operatorname{Spec}(k(\alpha)) \times_{\operatorname{Spec}(k)} X \rightarrow X
$$

is a $P$-torsor over $X$, and the homomorphism $\delta_{g}$ is induced by setting

$$
\delta_{g}(\alpha):=\left(X_{g}(\alpha)\right) \in \pi^{1}(X, P)
$$

Before starting the next result we recall that the group $W_{N}(k)_{F-1}$ is cyclic of order $p^{N}$. For each integer $i$ with $0 \leq i \leq N$ we let $P^{(i)}$ denote the subgroup of $P$ of order $p^{N-i}$.

LEMMA 2.7. Let $\alpha \in W_{N}(k)$ be a lift of a generator of the group $W_{N}(k)_{F-1}$, and fix an integer $i$ with $0 \leq i \leq N$. Then as $j$ varies over the set of integers which are coprime to $p$ the class $\delta_{g}\left(p^{i} j \alpha\right)$ varies over the set of elements of $\pi^{1}(X, P)$ which arise from the $P$-torsor associated to the constant $P^{(i)}$-cover $X_{i}$ of $X$ with respect to the different possible identifications of $\mathrm{Gal}\left(X_{i} / X\right)$ with $P^{(i)}$.

Proof. We write $V$ for the "Verschiebung" operator on Witt vectors. Thus, for any vector $a=\left(a_{0}, a_{1}, \ldots, a_{N-1}\right) \in W_{N}(k)$ one has $V a=\left(0, a_{0}, a_{1}, \ldots, a_{N-2}\right) \in$ $W_{N}(k)$. Using the identity $V \circ F=F \circ V=p$ (cf. [S1], Chapter II, §6) one can show that since $\alpha$ is a lift of a generator of $W_{N}(k)_{F-1}$ there exists a vector $\beta=\left(b_{0}, b_{1}, \ldots, b_{N-i-1}\right) \in W_{N-i}(k)$ with $b_{0} \in k \backslash(F \Leftrightarrow 1) k$ and which is such that $p^{i} \alpha=V^{i} \beta \in W_{N}(k)$. We recall that since $b_{0} \in k \backslash(F \Leftrightarrow 1) k$, the algebra $k(\beta)$ identifies with the unique field extension $k_{i}$ of $k$ which has group $P^{(i)}$ (cf. [L1], Chapter 8, Exercises 42-47).

We now consider the algebra $k\left(p^{i} \alpha\right)$. If $i=0$ then $\alpha=\beta$ so that $k(\alpha)=k(\beta)$ trivially. If $i \geq 1$ then from (2.2) (with $\alpha$ replaced by $p^{i} \alpha$ ) we deduce that

$$
F\left(X_{0}, X_{1}, \ldots, X_{i-1}\right) \Leftrightarrow\left(X_{0}, X_{1}, \ldots, X_{i-1}\right)=0 \quad \text { in } \quad W_{i}\left(k\left[X_{0}, X_{1}, \ldots, X_{i-1}\right]\right) .
$$


It follows that the image of the vector $\left(X_{0}, X_{1}, \ldots, X_{i-1}\right)$ in $W_{i}\left(k\left(p^{i} \alpha\right)\right)$ in fact lies in $W_{i}\left(\mathbb{F}_{p}\right) \subseteq W_{i}\left(k\left(p^{i} \alpha\right)\right)$. For each $\gamma \in W_{i}\left(\mathbb{F}_{p}\right)$ we let $k\left(p^{i} \alpha\right)_{\gamma}$ denote the $k$-algebra obtained from $k\left(p^{i} \alpha\right)$ by setting $\left(X_{0}, X_{1}, \ldots, X_{i-1}\right)=\gamma$; then there is a direct product decomposition $k\left(p^{i} \alpha\right)=\prod_{\gamma \in W_{i}\left(\mathbb{F}_{p}\right)} k\left(p^{i} \alpha\right)_{\gamma}$. With respect to the given action (2.3) the action of $g^{s}$ induces an isomorphism of $k$-algebras between $k\left(p^{i} \alpha\right)_{\gamma}$ and $k\left(p^{i} \alpha\right)_{\gamma+s}$. It follows that each $k$-algebra $k\left(p^{i} \alpha\right)_{\gamma}$ admits an action of $P^{(i)}=\left\langle g^{p^{i}}\right\rangle$, and is in fact isomorphic to $k\left(p^{i} \alpha\right)_{0}$ as a Galois $k\left[P^{(i)}\right]$-algebra. Now the relations defining $k\left(p^{i} \alpha\right)_{0}$ are equivalent to

$$
\begin{gathered}
F\left(0, \ldots, 0, X_{i}, X_{i+1}, \ldots, X_{N-1}\right) \Leftrightarrow\left(0, \ldots, 0, X_{i}, X_{i+1}, \ldots, X_{N-1}\right) \\
=p^{i} \alpha \text { in } W_{N}\left(k\left[X_{0}, X_{1}, \ldots, X_{N-1}\right]\right),
\end{gathered}
$$

that is, to

$$
\begin{gathered}
V^{i}\left(F\left(X_{i}, X_{i+1}, \ldots, X_{N-1}\right) \Leftrightarrow\left(X_{i}, X_{i+1}, \ldots, X_{N-1}\right) \Leftrightarrow \beta\right) \\
=0 \text { in } W_{N}\left(k\left[X_{0}, X_{1}, \ldots, X_{N-1}\right]\right)
\end{gathered}
$$

and since $V^{i}$ is injective this is equivalent to

$$
F\left(X_{i}, X_{i+1}, \ldots, X_{N-1}\right) \Leftrightarrow\left(X_{i}, X_{i+1}, \ldots, X_{N-1}\right)=\beta \quad \text { in } \quad W_{N-i}\left(k\left[X_{i}, \ldots, X_{N-1}\right]\right) .
$$

Each Galois $k\left[P^{(i)}\right]$-algebra $k\left(p^{i} \alpha\right)_{\gamma}$ is therefore isomorphic to $k(\beta)$.

At this stage we have shown that for each integer $i$ with $0 \leq i \leq N$ the algebra $k\left(p^{i} \alpha\right)$ is isomorphic to the Galois $P$-algebra over $k$ which has splitting field the unique extension $k_{i}$ of $k$ of group $P^{(i)}$ (with respect to some identification of $\mathrm{Gal}\left(k_{i} / k\right)$ with $\left.P^{(i)}\right)$. To conclude the proof of Lemma 2.7 we now merely note that if $j$ is coprime to $p$ then the map

$$
\left(X_{0}, X_{1}, \ldots, X_{N-1}\right) \mapsto j\left(X_{0}, X_{1}, \ldots X_{N_{1}}\right) \quad \text { in } \quad W_{N}\left(k\left[X_{0}, X_{1}, \ldots, X_{N-1}\right]\right)
$$

induces an isomorphism of Galois $k[P]$-algebras from $k\left(p^{i} \alpha\right)$ to the algebra which as a $k$-algebra is equal to $k\left(p^{i} j \alpha\right)$, but has a $P$-action obtained by composing the action (2.3) with the automorphism $g \mapsto g^{j}$ of $P$.

The equality Image $\left(\delta_{g}\right)=\operatorname{ker}\left(\psi_{P}\right)$ is an immediate consequence of Lemmas 2.6 and 2.7, and so we have now completed the proof of Theorem 2.5.

Taken together with the isomorphism (1.2) (with $G=P$ ) the results of Proposition 2.4 and Theorem 2.5 give a commutative diagram in which both the row and column involving $\pi^{1}(X, P)$ are exact (and in which the map $\lambda_{g}$ is defined by 
the requirement that the diagram should commute):

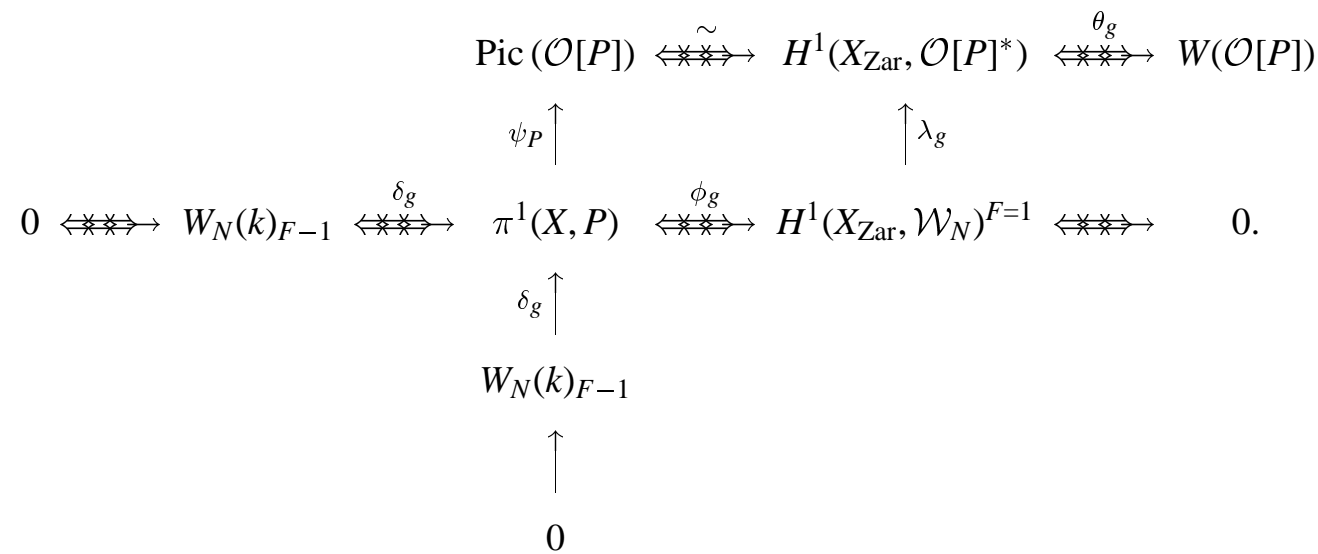

Since $\theta_{g}$ is an isomorphism it is clear from this diagram that $\Re_{X}^{0}(P)\left(=\operatorname{Image}\left(\psi_{P}\right)\right)$ is in general much smaller than $\operatorname{Pic}(\mathcal{O}[P])$. By using the description of the map $\phi_{g}$ given in ([Mi], p. 127) (after identifying $P$ with $\mathbb{Z} / p^{N}$ via the chosen generator $g$ ) it is in fact not too difficult to describe the map $\lambda_{g}$ explicitly. However, it would seem to be more difficult to describe the composite map $\theta_{g} \circ \lambda_{g}$ in terms of natural operations on Witt vectors. (Partial results in this direction are discussed in [A, $\mathrm{Bu}]$ ).

2.2. Tame covers of degree prime to $p$. We now turn to consider the Galois $H$-cover $f^{p}: Y(p) \rightarrow X$. In this section we shall state realizability results analogous to those of $[\mathrm{M}]$ and $[\mathrm{Bu}]$. To do this we shall need some further notation (and we follow that of $[\mathrm{M}]$ ).

Recalling that $\hat{H}:=\operatorname{Hom}\left(H,\left(k^{c}\right)^{*}\right)$, we define a homomorphism det: $R_{H} \rightarrow \hat{H}$ by setting

$$
\operatorname{det}\left(\sum_{\chi \in \hat{H}} a_{\chi} \chi\right)=\prod_{\chi \in \hat{H}} \chi^{a_{\chi}} \quad\left(a_{\chi} \in \mathbb{Z}\right)
$$

With $A_{\hat{H}}$ denoting the kernel of det there is a natural restriction map

$$
\operatorname{Rag}_{H}: \operatorname{Hom}_{\Omega_{K}}\left(R_{H}, \operatorname{Pic}\left(K^{\prime}\right)\right) \rightarrow \operatorname{Hom}_{\Omega_{K}}\left(A_{\hat{H}}, \operatorname{Pic}\left(K^{\prime}\right)\right)
$$

We let $H(\Leftrightarrow 1)$ denote the group $H$ which is endowed with a structure as $\Omega_{K^{-}}$ module via the inverse cyclotomic character. We choose a root of unity $\mu$ in $k^{c}$ of order equal to the exponent of $H$. Following McCulloh [M] we shall in $\S 5$ define a natural $\Omega_{K}$-equivariant "Stickelberger type" map $\Theta_{H, \mu}: A_{\hat{H}} \rightarrow \mathbb{Z}[H(\Leftrightarrow 1)]$, the 
transpose of which gives rise to a map

$$
\Theta_{H, \mu}^{t}: \operatorname{Hom}_{\Omega_{K}}\left(\mathbb{Z}[H(\Leftrightarrow 1)], \operatorname{Pic}\left(K^{\prime}\right)\right) \rightarrow \operatorname{Hom}_{\Omega_{K}}\left(A_{\hat{H}}, \operatorname{Pic}\left(K^{\prime}\right)\right) .
$$

For each $\Omega_{K}$-orbit $T$ of $H(\Leftrightarrow 1)$ we let $K_{T}$ denote the (constant) field extension of $K$ which corresponds by Galois theory to the pointwise stabilizer of $T$ in $\Omega_{K}$. We fix an isomorphism

$$
\operatorname{Hom}_{\Omega_{K}}\left(\mathbb{Z}[H(\Leftrightarrow 1)], \operatorname{Pic}\left(K^{\prime}\right)\right) \cong \bigoplus_{T \in \Omega_{K} \backslash H(-1)} \operatorname{Pic}\left(K_{T}\right) .
$$

(The precise choice of this isomorphism doesn't matter in what follows.) We define a "prime element" of $\bigoplus_{T \in \Omega_{K} \backslash H(-1)} \operatorname{Pic}\left(K_{T}\right)$ to be any element which is nonzero in precisely one component, that corresponding to $T_{0} \in \Omega_{K} \backslash H(\Leftrightarrow 1)$ say, and in this component is the class of a prime divisor of $K_{T_{0}}$ which is of relative degree one in $K_{T_{0}} / K$. We let $F_{X, H}$ denote the subset of $\bigoplus_{T \in \Omega_{K} \backslash H(-1)} \operatorname{Pic}\left(K_{T}\right)$ which consists of arbitrary finite sums of prime elements which lie over distinct prime divisors of $K$.

If $Y$ is a smooth projective curve with constant field $l$ then for each point $y \in Y_{0}$ we let $l_{y}$ denote the residue field of the stalk $\mathcal{O}_{Y, y}$ and we let $d_{y}$ denote the positive integer defined by $\# l_{y}=\# l^{d_{y}}$. The degree of a divisor $D=\sum_{y \in Y_{0}} n_{y} y \in$ $\operatorname{Div}(L)$ is defined to be $\operatorname{deg}_{L}(D):=\sum_{y \in Y_{0}} n_{y} d_{y}$, and we call \# $l^{\operatorname{deg}_{L}(D)}$ the "norm" of the divisor $D$.

For any strictly positive integer $N$ and function field $L$ we let $\operatorname{Div}^{N}(L)$ denote the subgroup of $\operatorname{Div}(L)$ given by those divisors whose norm is congruent to 1 modulo $N$, and we write $\operatorname{Pic}^{N}(L)$ for the image of $\operatorname{Div}^{N}(L)$ in $\operatorname{Pic}(L)$. We let $\operatorname{Pic}^{N}(\mathcal{O}[H])$ denote the subgroup of $\mathrm{Pic}(\mathcal{O}[H])$ consisting of those elements whose image under the isomorphism $\theta_{\mathcal{O}[H]}$ (cf. Proposition 1.3) can be represented by homomorphisms which are valued entirely above $\operatorname{Pic}^{N}(K)$. We let $\mathcal{C}(H)$ denote the set of cyclic subgroups of $H$.

In addition to the above notation we let $\Re_{X, 0}^{0}(H)$, respectively $\Re_{X}^{0}(H)$, denote the subset of Pic $(\mathcal{O}[H])$ given by the classes of structure sheaves in all étale, respectively tame, Galois $H$-covers of $X$. We write $\Re_{X, 0}(H)$, respectively $\Re_{X}(H)$, for the subgroup of $\mathrm{Pic}(\mathcal{O}[H])$ which is generated by the classes of arbitrary $H$ stable invertible sheaves arising from étale, respectively tame, Galois $H$-covers of $X$. In $\S 5$ and $\S 6$ we shall prove the following theorem.

THEOREM 2.8.

(i) $\Re_{X, 0}^{0}(H)=\operatorname{kernel}\left(\operatorname{Rag}_{H}\right)$.

(ii) $\Re_{X}^{0}(H)=\operatorname{Rag}_{H}^{-1}\left(\Theta_{H, \mu}^{t}\left(F_{X, H}\right)\right)$.

(iii) $\Re_{X, 0}(H)=\Re_{X, 0}^{0}(H)+\operatorname{ind}_{\{1\}}^{H} \operatorname{Pic}(\mathcal{O})$.

(iv) $\Re_{X}(H)=\left\langle\Re_{X}^{0}(H)\right\rangle+\sum_{C \in \mathcal{C}(H)} \operatorname{ind}_{C}^{H} \operatorname{Pic}^{\# C}(\mathcal{O}[C])$. 
Remarks 2.10. (i) We shall see in $\S 5$ that the subgroup $\Theta_{H, \mu}^{t}\left(F_{X, H}\right)$ which occurs in statement (ii) is independent of the choice of $\mu$.

(ii) The above description of $\Re_{X}^{0}(H)$ is a geometric analogue of McCulloh's description of the realizable classes arising from relative abelian extensions of number fields (cf. [M]). The geometric interest of such analogues is pointed out by Chinburg in ([Ch2], Introduction). The description of $\Re_{X}(H)$ is an analogue of the main result of $[\mathrm{Bu}]$.

(iii) It follows from this description that $\Re_{X, 0}^{0}(H)$ is always a subgroup (this can also be seen via the approach of $\S 2.1)$, whilst $\Re_{X}^{0}(H)$ is in general not a subgroup (cf. Remark 5.7).

3. Realizable Euler characteristic classes. Let now $X$ be any scheme which is proper and of finite type over a Noetherian ring $A$. In [Ch1] Chinburg has shown how to attach to each $\mathcal{O}_{X}[G]$-vector bundle $\mathcal{V}$ a natural Euler characteristic class $\chi R \Gamma_{G}(\mathcal{V})$ in the Grothendieck group $C T(A[G])$ of the category of finite generated $A[G]$-modules which are cohomologically trivial for $G$ (see also [Ch2], [Ch,E]). In particular, if the order of $G$ is coprime to the residue characteristic of each $\mathcal{P} \in \operatorname{Spec}(\mathrm{A})$ then $\chi R \Gamma_{G}(\mathcal{V})$ coincides with the usual Lefschetz trace $L_{X, G}(\mathcal{V}):=\sum_{i \geq 0}(\Leftrightarrow 1)^{i}\left(H^{i}(X, \mathcal{V})\right) \in C T(A[G])=G_{0}(A[G])$. More generally, use of the Cech hypercohomology complex of $\mathcal{V}$ (with respect to any open affine cover of $X$ ) gives one an algorithm for computing $\chi R \Gamma_{G}(\mathcal{V})$. For example, if $X$ is a smooth projective curve over a finite field $A=k$, and $\mathcal{L}$ is a $G$-stable Weil divisor on a tame Galois $G$-cover $f: Y \rightarrow X$ then Chinburg has in this way given the following more explicit description of $\chi R \Gamma_{G}\left(f_{*} \mathcal{L}\right)$ (cf. [Ch1], Theorem 2.18).

Choose any nonempty affine open subset $U$ of $X$. Then there exists an element $\beta \in K_{Y}$ such that $\mathcal{O}(U)[G] \beta$ has finite index in $f_{*} \mathcal{L}(U)$, and for each $x \in X \backslash U$ the stalk of $f_{*} \mathcal{L}$ at $x$ has finite index in $\mathcal{O}_{x}[G] \beta$. Writing $g_{Y}$ for the genus of $Y$ then for each such choice of element $\beta$ one has an equality

$$
\chi R \Gamma_{G}\left(f_{*} \mathcal{L}\right)=\left(1 \Leftrightarrow g_{Y}\right)(k[G])+\left(\frac{f_{*} \mathcal{L}(U)}{\mathcal{O}(U)[G] \beta}\right) \Leftrightarrow \sum_{x \in X \backslash U}\left(\frac{\mathcal{O}_{x}[G] \beta}{f_{*} \mathcal{L}_{x}}\right),
$$

in $C T(k[G])=K_{0}(k[G])$.

In general, the map $\mathcal{V} \mapsto \chi R \Gamma_{G}(\mathcal{V})$ induces a homomorphism $\chi R \Gamma_{G}$ : $K_{0}(\mathcal{O}[G]) \rightarrow C T(A[G])$ (cf. [Ch2], Remark 2.7). Moreover, from ([Ch2], Remark 2.8) one knows that $\chi R \Gamma_{G}(\mathcal{O}[G])=\chi(\mathcal{O})(A[G])$ (where $\chi(\mathcal{O})$ denotes the Euler characteristic of $\mathcal{O}$ ) and hence one has a stable Euler characteristic homomorphism $\tilde{\chi} R \Gamma_{G}: \mathrm{Cl}(\mathcal{O}[G]) \rightarrow C T(A[G]) /(A[G])$. (Similarly, if the order of $G$ is coprime to the residue characteristic of each $\wp \in \operatorname{Spec}(A)$, then we write $\tilde{L}_{X, G}: \mathrm{Cl}(\mathcal{O}[G]) \rightarrow C T(A[G]) /(A[G])$ for the stable Lefschetz trace homomorphism.) In this section we use the results of $\S 2$ to describe explicitly the subgroup $\tilde{\chi} R \Gamma_{G}\left(\Re_{X}(G)\right) \subseteq \mathrm{Cl}(k[G])$ of realizable Euler characteristic classes associated to a smooth projective curve $X$ which has finite constant field $k$. 
Identifying $H$ with the quotient $G / P$ there is an isomorphism $\phi_{H}^{G}: \mathrm{Cl}(k[H]) \rightarrow$ $\mathrm{Cl}(k[G])$ which is induced by inflating modules from $H$ to $G$ and then taking projective envelopes over $G$ (cf. [S3], III, §1.4). On the other hand, given any étale Galois $P$-cover $e: W \rightarrow X$ of $X$ one can construct a natural homomorphism $\theta_{e}: \Re_{X}(H) \rightarrow \Re_{X}(G)$ in the following way. If $f: Y \rightarrow X$ is any Galois $H$-cover of $X, \mathcal{L}$ is a $H$-stable invertible $\mathcal{O}_{Y}$-sheaf, and $h: Z \rightarrow X$ is the tame $G$-cover of $X$ which has function field equal to the compositum of $K_{W}$ and $K_{Y}$, then Lemma 2.1 implies that $f_{*} \mathcal{L} \otimes_{\mathcal{O}} e_{*} \mathcal{O}_{W}$ is $\mathcal{O}[G]$-isomorphic to $h_{*} \mathcal{M}$ for some $G$-stable invertible $\mathcal{O}_{Z}$-sheaf $\mathcal{M}$. The assignment $\left(f_{*} \mathcal{L}\right) \mapsto\left(f_{*} \mathcal{L} \otimes_{\mathcal{O}} e_{*} \mathcal{O}_{W}\right)$ thus gives rise to a well-defined homomorphism $\theta_{e}$ from $\Re_{X}(H)$ to $\Re_{X}(G)$.

For any commutative ring $A$ we let $S_{A}$ denote the set of isomorphism classes of finitely generated simple $A$-modules.

Lemma 3.1. For each étale Galois P-cover $e: W \rightarrow X$ of $X$ the following diagram commutes:

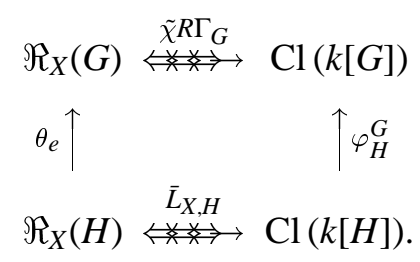

Proof. Let $f: Y \rightarrow X$ be a Galois $H$-cover of $X$, and let $h: Z \rightarrow X$ be the corresponding tame Galois $G$-cover of $X$, described above. We let $\mathcal{O}_{1}$ and $\mathcal{O}_{2}$ denote the structure sheaves of $Y$ and $W$ respectively. For each $H$-stable invertible $\mathcal{O}_{1}$-sheaf $\mathcal{L}_{1}$ we wish to prove the following equality in $\mathrm{Cl}(k[G])$ :

$$
\tilde{\chi} R \Gamma_{G}\left(f_{*} \mathcal{L}_{1} \otimes_{\mathcal{O}} e_{*} \mathcal{O}_{2}\right)=\varphi_{H}^{G} \circ \tilde{L}_{X, H}\left(f_{*} \mathcal{L}_{1}\right)
$$

To do this we choose a closed point $x_{0} \in X$ and let $U$ denote the affine open set $X \backslash\left\{x_{0}\right\}$. By Lemma 2.2 we may choose $\beta_{2} \in e_{*} \mathcal{O}_{2}(U)$ such that $e_{*} \mathcal{O}_{2}(U)=$ $\mathcal{O}(U)[P] \beta_{2}$. Note in particular that $\sum_{g \in P} g \beta_{2} \in \mathcal{O}(U)^{*}=k^{*}$. Choosing an element $\alpha_{2} \in K$ which has a pole of sufficiently high order at $x_{0}$ and has no poles on $U$ we can ensure that $\alpha_{2} \beta_{2} \in e_{*} \mathcal{O}_{2}(U)$ and $e_{*} \mathcal{O}_{2, x_{0}} \subseteq \mathcal{O}_{x_{0}}[P] \alpha_{2} \beta_{2}$. We also choose a free generator $\beta_{2}^{\prime}$ of the stalk $e_{*} \mathcal{O}_{2, x_{0}}$ over $\mathcal{O}_{x_{0}}[P]$, and let $\gamma_{2} \in \mathcal{O}_{x_{o}}[P]$ be defined by $\beta_{2}^{\prime}=\lambda_{2} \alpha_{2} \beta_{2}$. Note that since both $\sum_{g \in P} g \beta_{2}$ and $\sum_{g \in P} g \beta_{2}^{\prime}$ are units at $x_{0}$ the augmentation $\epsilon\left(\lambda_{2}\right)$ of $\lambda_{2}$ has the same $x_{0}$-valuation as does $\alpha_{2}^{-1}$.

Choosing now an element $\beta_{1} \in f_{*} \mathcal{L}_{1}(U)$ such that both $\mathcal{O}(U)[H] \beta_{1}$ has finite index in $f_{*} \mathcal{L}_{1}(U)$ and $f_{*} \mathcal{L}_{1, x_{0}}$ has finite index in $\mathcal{O}_{x_{0}}[H] \beta_{1}$, we shall compute the Euler characteristic $\tilde{\chi} R \Gamma_{G}\left(f_{*} \mathcal{L}_{1} \otimes_{\mathcal{O}} e_{*} \mathcal{O}_{2}\right)$ via the formula (3.1) with respect to the element $\beta_{1} \otimes_{K} \alpha_{2} \beta_{2} \in K_{Z}^{*}$. In this way, one checks that $\tilde{\chi} R \Gamma_{G}\left(f_{*} \mathcal{L}_{1} \otimes_{\mathcal{O}} e_{*} \mathcal{O}_{2}\right)$ 
is equal to

$$
\begin{aligned}
& \left(\frac{f_{*} \mathcal{L}_{1}(U) \otimes_{\mathcal{O}(U)} e_{*} \mathcal{O}_{2}(U)}{\mathcal{O}(U)[H] \beta_{1} \otimes_{\mathcal{O}(U)} \mathcal{O}(U)[P] \alpha_{2} \beta_{2}}\right) \Leftrightarrow\left(\frac{\mathcal{O}_{x_{0}}[H] \beta_{1} \otimes_{\mathcal{O}_{x_{0}}} \mathcal{O}_{x_{0}}[P] \alpha_{2} \beta_{2}}{f_{*} \mathcal{L}_{1, x_{0}} \otimes_{\mathcal{O}_{x_{0}}} e_{*} \mathcal{O}_{2, x_{0}}}\right) \\
& =\left(\frac{f_{*} \mathcal{L}_{1}(U) \otimes \mathcal{O}(U) \mathcal{O}(U)[P] \beta_{2}}{\mathcal{O}(U)[H] \beta_{1} \alpha_{2} \otimes_{\mathcal{O}(U)} \mathcal{O}(U)[P] \beta_{2}}\right) \Leftrightarrow\left(\frac{\mathcal{O}_{x_{0}}[H] \beta_{1} \otimes_{\mathcal{O}_{x_{0}}} \mathcal{O}_{x_{0}}[P] \alpha_{2} \beta_{2}}{f_{*} \mathcal{L}_{1, x_{0}} \otimes_{\mathcal{O}_{x_{0}}} \mathcal{O}_{x_{0}}[P] \lambda_{2} \alpha_{2} \beta_{2}}\right) \\
& =\left(\frac{f_{*} \mathcal{L}_{1}(U) \otimes_{\mathcal{O}(U)} \mathcal{O}(U)[P]}{\mathcal{O}(U)[H] \beta_{1} \alpha_{2} \otimes_{\mathcal{O}(U)} \mathcal{O}(U)[P]}\right) \Leftrightarrow\left(\frac{\mathcal{O}_{x_{0}}[H] \beta_{1} \otimes_{\mathcal{O}_{x_{0}}} \mathcal{O}_{x_{0}}[P]}{f_{*} \mathcal{L}_{1, x_{0}} \otimes_{\mathcal{O}_{x_{0}}} \mathcal{O}_{x_{0}}[P] \lambda_{2}}\right) \\
& =\left(\frac{f_{*} \mathcal{L}_{1}(U)}{\mathcal{O}(U)[H] \beta_{1} \alpha_{2}} \otimes_{k} k[P]\right) \Leftrightarrow\left(\frac{\mathcal{O}_{x_{0}}[H] \beta_{1} \otimes_{\mathcal{O}_{x_{0}}} \mathcal{O}_{x_{0}}[P]}{f_{*} \mathcal{L}_{1, x_{0}} \otimes_{\mathcal{O}_{x_{0}}} \mathcal{O}_{x_{0}}[P] \lambda_{2}}\right) .
\end{aligned}
$$

Recalling that $\tilde{L}_{X, H}(\Leftrightarrow)=\tilde{\chi} R \Gamma_{H}(\Leftrightarrow)$ we can again apply (3.1) (this time with $G$ replaced by $H$, and the element $\beta$ taken to be $\alpha_{2} \beta_{1}$ ) in order to compute the right-hand side of (3.2). In this way one sees that the equality (3.2) follows from (3.3) if one has in $\mathrm{Cl}(k[G])$ an equality

$$
\left(\frac{\mathcal{O}_{x_{0}}[H] \beta_{1} \otimes_{\mathcal{O}_{x_{0}}} \mathcal{O}_{x_{0}}[P]}{f_{*} \mathcal{L}_{1, x_{0}} \otimes_{\mathcal{O}_{x_{0}}} \mathcal{O}_{x_{0}}[P] \lambda_{2}}\right)=\left(\frac{\mathcal{O}_{x_{0}}[H] \beta_{1} \alpha_{2}}{f_{*} \mathcal{L}_{1, x_{0}}} \otimes_{k} k[P]\right)
$$

Note that (3.4) would in turn follow if we could show that the left-hand side class of (3.4) considered as a function of $\lambda_{2} \in \mathcal{O}_{x_{0}}[P]$ depended only upon its augmentation $\epsilon\left(\lambda_{2}\right)$. Indeed, if this is the case then we may in (3.4) replace $\lambda_{2}$ by $\alpha_{2}^{-1}$ and in this case the equality of (3.4) is straightforward to verify.

We now write $\mathcal{O}$ for $\mathcal{O}_{x_{0}}$, and $\Lambda$, respectively $\Lambda_{1}$, for the group ring $\mathcal{O}[G]$, respectively $\mathcal{O}[P]$. For each element $\mu \in \mathcal{O}[H]^{*}$ we consider the function $c_{\mu}: \Lambda_{1}^{*} \rightarrow$ $\mathrm{Cl}(k[G])$ obtained by defining $c_{\mu}\left(\lambda_{1}\right)$ for each $\lambda_{1} \in \Lambda_{1}^{*}$ to be the class of the $k[G]$-module $C_{\mu}\left(\lambda_{1}\right):=\left(\Lambda / \Lambda\left(\mu \otimes \lambda_{1}\right)\right)$. Our proof of (3.2) is completed by the next result.

Sub-Lemma 3.2. For each choice of $\mu \in \mathcal{O}[H]^{*}$ the function $c_{\mu}$ factors through the augmentation homomorphism $\epsilon: \Lambda_{1}^{*} \rightarrow \mathcal{O}^{*}$.

Proof. For each $M \in S_{k[G]}$ we let $P_{M}$ denote its projective envelope. For each such $M$ we define an integer $c_{\mu, M}\left(\lambda_{1}\right)$ by the isomorphism $C_{\mu}\left(\lambda_{1}\right) \cong$ $\oplus_{M \in S_{k[G]}} c_{\mu, M}\left(\lambda_{1}\right) P_{M}$. The key point in proving this sub-lemma is to compute each of the integers $c_{\mu, M}\left(\lambda_{1}\right)$ by taking into account the fact that each $M$ is the inflation from $H=G / P$ to $G$ of a simple $k[H]$-module $M^{\prime}$. To do this we let Jac $(k[G])$ denote the Jacobson radical of $k[G]$. The quotient $k[G] / \operatorname{Jac}(k[G])$ identifies with the semisimple ring $\oplus_{M \in S_{k[G]}} k_{M}$ where here $k_{M}$ is the finite field extension of $k$ of cardinality $\mathbb{H}_{\operatorname{Hom}_{G}}(M, M)$. The module $M$ corresponds to a character $\chi_{M}: G \rightarrow k_{M}^{*}$ the restriction to $P$ of which is trivial. Note that $C_{\mu}\left(\lambda_{1}\right) / \operatorname{Jac}(k[G]) C_{\mu}\left(\lambda_{1}\right) \cong$ 
$\bigoplus_{M \in S_{k[G]}} c_{\mu, M}\left(\lambda_{1}\right) M$. But, setting $\tilde{\Lambda}:=\Lambda / \operatorname{Jac}(k[G]) \Lambda$, one has

$C_{\mu}\left(\lambda_{1}\right) / \operatorname{Jac}(k G) C_{\mu}\left(\lambda_{1}\right) \cong \tilde{\Lambda} / \tilde{\Lambda}\left(\mu \otimes \lambda_{1}\right) \cong \bigoplus_{M \in S_{k[G]}}\left(\mathcal{O} \otimes_{k} k_{M} / \chi_{M}\left(\mu \otimes \lambda_{1}\right) \mathcal{O} \otimes_{k} k_{M}\right)$,

and so since $\chi_{M}\left(\mu \otimes \lambda_{1}\right)=\chi_{M^{\prime}}(\mu) \epsilon\left(\lambda_{1}\right)$ the result is clear.

From Lemmas 2.1 and 3.1 one has

$$
\tilde{\chi} R \Gamma_{G}\left(\Re_{X}(G)\right)=\tilde{\chi} R \Gamma_{G}\left(\sum_{e: W \rightarrow X} \theta_{e} \Re_{X}(H)\right)=\varphi_{H}^{G} \tilde{L}_{X, H}\left(\Re_{X}(H)\right)
$$

(with the sum taken over all étale $P$-covers $e: W \rightarrow X$ of $X$ ) and so we are reduced to considering realizability issues for Galois $H$-covers of $X$. In this semisimple setting we must give a Hom-description of the reduced Lefschetz trace. This is possible as a consequence of the Riemann-Roch theorem for line bundles on $X$. To be more specific we need a little more notation.

We fix an algebraic closure $\mathbb{Q}_{p}^{c}$ of $\mathbb{Q}_{p}$. For any finite field $k$ of characteristic $p$ we let $W(k)$ denote the Witt ring of $k$ (that is, the unique absolutely unramified characteristic 0 local ring with residue field $k$ (so that $\left.W(k) \subset \mathbb{Q}_{p}^{c}\right)$ ), and we write $K(k)$ for the field of fractions of $W(k)$. For any finite group $I$ we set $I^{\dagger}=\operatorname{Hom}\left(I, \mathbb{Q}_{p}^{c *}\right)$, and we let $R_{I, p}$ denote the ring of $\mathbb{Q}_{p}^{c}$-valued characters of $I$. For any field extension $F$ of $K(k)$ in $\mathbb{Q}_{p}^{c}$ we set $\Omega_{F}=\operatorname{Gal}\left(\mathbb{Q}_{p}^{c} / F\right)$. For any choice of uniformizing parameter $\pi_{F}$ of $F$ there is a natural injection

$$
D_{k, F, I, \pi_{F}}: K_{0}(K[I]) \hookrightarrow \operatorname{Hom}_{\Omega_{F}}\left(R_{I, p}, \mathbb{Q}_{p}^{c}\right)
$$

which is defined in the following way: if $\bar{P}$ is a projective $k[I]$-module then there is a projective $W(k)[I]$-module $P$ which is unique up to isomorphism and is such that $P \otimes_{W(k)} k \cong \bar{P}$. If $P \otimes_{W(k)} \mathbb{Q}_{p}^{c}$ has character $\sum_{\chi \in I^{\dagger}} m_{\chi} \chi$ then $D_{k, F, I, \pi_{F}}(\chi)=\pi_{F}^{-m_{\chi}}$. In the case $k=\mathbb{F}_{p}$ and $F=\mathbb{Q}_{p}$ the injection $D_{\mathbb{F}_{p}, \mathbb{Q}_{p}, I, p}$ was introduced by Chinburg in order to relate de Rham structure invariants to invariants of functional equations of L-functions (cf. [Ch2], §4). Letting $\tilde{H o m}_{\Omega_{F}}\left(R_{I, p}, \mathbb{Q}_{p}^{c *}\right)$ denote the quotient of $\operatorname{Hom}_{\Omega_{F}}\left(R_{I, p}, \mathbb{Q}_{p}^{c *}\right)$ by the subgroup generated by $D_{k, F, I, \pi_{F}}(k[I])$ there is induced a natural injection

$$
\tilde{D}_{k, F, I, \pi_{F}}: \mathrm{Cl}(k[I]) \hookrightarrow \tilde{H}_{\Omega_{F}}\left(R_{I, p}, \mathbb{Q}_{p}^{c *}\right)
$$

We first note that the homomorphism $\varphi_{H}^{G}$ is easily described in terms of these Hom-descriptions. 
LEMMA 3.3. For each choice of $F$ and $\pi_{F}$ as above the following square commutes:

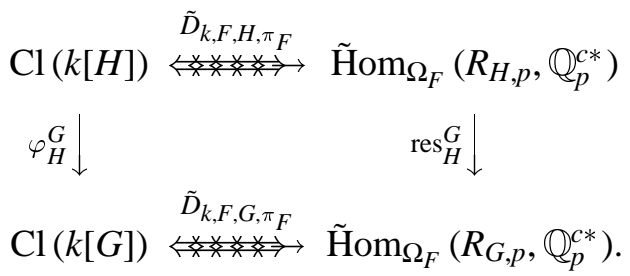

Proof. The homomorphism $\varphi_{H}^{G}$ sends the class of a projective $k[H]$-module $\bar{M}$ to the class of the projective $k[G]$-module $k[P] \otimes_{k} \bar{M}$. In addition, if $M$ is any projective $W(k)[H]$-module satisfying $M \otimes_{W(k)} k \cong \bar{M}$ then $M_{G}:=W(k)[P] \otimes_{W(k)} M$ is a projective $W(k)[G]$-module satisfying $M_{G} \otimes_{W(k)} k \cong k[P] \otimes_{k} \bar{M}$. We now write $\langle\Leftrightarrow, \Leftrightarrow\rangle_{G}$ and $\langle\Leftrightarrow, \Leftrightarrow\rangle_{H}$ for the standard pairings on the character rings $R_{G, p}$ and $R_{H, p}$ respectively. Applying Frobenius reciprocity we obtain for each character $\psi \in R_{G, p}$ the equalities

$$
\left\langle\chi_{M_{G}}, \psi\right\rangle_{G}=\left\langle\operatorname{ind}_{H}^{G} \chi_{M}, \psi\right\rangle_{G}=\left\langle\chi_{M}, \operatorname{res}_{H}^{G} \psi\right\rangle_{H}
$$

and this in turn implies the commutativity of the above square.

We shall now give a Hom-description of the Lefschetz trace $\tilde{L}_{X, H}$. To do this we first note that, since $p \nmid \# H$, the groups $I^{\dagger}$ and $\hat{I}\left(=\operatorname{Hom}\left(I,\left(k^{c}\right)^{*}\right)\right)$ correspond bijectively. For each element $\phi \in I^{\dagger}$ we write $\hat{\phi}$ for the corresponding element of $\hat{I}$, and we let $K(\phi)$ denote the field extension of $K$ obtained by adjoining the values of $\hat{\phi}$. We define a homomorphism

$$
D_{X, H}: \operatorname{Hom}_{\Omega_{K}}\left(R_{H}, \operatorname{Pic}\left(K^{\prime}\right)\right) \rightarrow \tilde{\operatorname{Hom}} \Omega_{K(k)}\left(R_{H, p}, \mathbb{Q}_{p}^{c *}\right)
$$

by setting $D_{X, H}(h)(\phi)=\pi_{K(k)}^{\operatorname{deg}_{K(\phi)} h(\tilde{\phi})}$ for each $h \in \operatorname{Hom}_{\Omega_{K}}\left(R_{H}, \operatorname{Pic}\left(K^{\prime}\right)\right)$ and $\phi \in I^{\dagger}$.

Proposition 3.4. Set $F=K(k)$. Then the following square commutes:

$$
\begin{array}{ccc}
\operatorname{Pic}(\mathcal{O}[H]) & \stackrel{\tilde{L}_{X, H}}{\longrightarrow} & \mathrm{Cl}(k[H]) \\
\theta_{\mathcal{O}[H]} \downarrow & \tilde{D}_{k, F, H, \pi} \downarrow \\
\operatorname{Hom}_{\Omega_{K}}\left(R_{H}, \operatorname{Pic}\left(K^{\prime}\right)\right) & \stackrel{D_{X, H}}{\longrightarrow} & \tilde{H o m}_{\Omega_{F}}\left(R_{H, p}, \mathbb{Q}_{p}^{c *}\right) .
\end{array}
$$

Proof. We write $\chi(\mathcal{L})$ for the Euler characteristic of a locally-free $\mathcal{O}$-sheaf $\mathcal{L}$, and for each $k[H]$-module $M$ we write $M^{*}$ for the dual module $\operatorname{Hom}_{k}(M, k)$. For each invertible $\mathcal{O}[H]$-module $\mathcal{L}$ and each simple module $V \in S_{k[H]}$ we let $\mathcal{L}_{V}$ 
denote the $\mathcal{O}$-sheaf $H^{0}\left(H, \mathcal{L} \otimes_{k} V\right)$. This sheaf is locally-free of rank $\operatorname{dim}_{k}(V)$, and from the argument of ([E,L], Lemma 2.2) one has

$$
L_{X, H}(\mathcal{L})=\sum_{V \in S_{k[H]}}\left(\chi\left(\mathcal{L}_{V^{*}}\right) / \operatorname{dim}_{k}(V)\right)[V] \in G_{0}(k[H])
$$

For each character $\phi \in I^{\dagger}$ it follows that

$$
\begin{aligned}
\tilde{D}_{k, F, H, \pi_{F}} \tilde{L}_{X, H}(\mathcal{L})(\phi) & =\tilde{D}_{k, G, H, \pi} \tilde{L}_{X, H}\left(\mathcal{L} \otimes_{\mathcal{O}[H]} \mathcal{F}^{-1}\right)(\phi) \\
& =\pi_{F}^{\left(\chi\left(\mathcal{F}_{V^{*}}\right)-\chi\left(\mathcal{L}_{V^{*}}\right)\right) / \operatorname{dim}_{k}(V)}
\end{aligned}
$$

where here $\mathcal{F}$ is any free rank one $\mathcal{O}[H]$-module and $\hat{\phi}$ occurs in the character of the simple $k[H]$-module $V$. We now fix a character $\phi \in I^{\dagger}$, and let $V$ be the corresponding simple $k[H]$-module (as in (3.8)). We let $k^{\prime}$ denote the field extension of $k$ which is generated by the values of $\hat{\phi}$. Thus we have $K k^{\prime}=K(\phi)$. We set $X^{\prime}=X \times_{k} k^{\prime}, \mathcal{O}^{\prime}=\mathcal{O}_{X^{\prime}}$, and for any $\mathcal{O}$-sheaf $\mathcal{M}$ we let $\mathcal{M}^{\prime}$ denote the corresponding $\mathcal{O}^{\prime}$-sheaf $\mathcal{M} \otimes_{k} k^{\prime}$ obtained by base-extension.

For any locally-free $\mathcal{O}$-sheaf $\mathcal{M}$ the Riemann-Roch theorem (cf. [H], Chapter $4, \S 1$ or [We], Chapter VI) gives an equality

$$
\chi\left(\mathcal{M}_{V^{*}}\right)=\operatorname{deg}_{K}\left(\mathcal{M}_{V^{*}}\right)+\operatorname{rank}_{\mathcal{O}}\left(\mathcal{M}_{V^{*}}\left(1 \Leftrightarrow g_{X}\right)\right)
$$

where here $g_{X}$ is the genus of $X$, and hence one has

$$
\begin{aligned}
\chi\left(\mathcal{F}_{V^{*}}\right) \Leftrightarrow \chi\left(\mathcal{L}_{V^{*}}\right) & =\operatorname{deg}_{K}\left(\mathcal{F}_{V^{*}}\right) \Leftrightarrow \operatorname{deg}_{K}\left(\mathcal{L}_{V^{*}}\right) \\
& =\operatorname{deg}_{K(\phi)}\left(\mathcal{F}_{V^{*}}^{\prime}\right) \Leftrightarrow \operatorname{deg}_{K(\phi)}\left(\mathcal{L}_{V^{*}}^{\prime}\right) .
\end{aligned}
$$

For each character $\kappa \in \hat{I}$ which occurs in $V$ we let $V_{\kappa}$ denote the corresponding representation of $H$ over $k^{\prime}$, and we let $e_{\kappa}$ denote the primitive idempotent $(\# H)^{-1} \sum_{h \in H} \kappa\left(h^{-1}\right) h$ of $k^{\prime}[H]$. We may suppose that $\mathcal{F}$ and $\mathcal{L}$ have the same generic stalk. We let $\ell_{0} \in \mathcal{F}(X)$ be a generator of $\mathcal{F}$ over $\mathcal{O}[H]$, and for each $x \in X_{0}$ we let $\ell_{x}$ be generator of the stalk $\mathcal{L}_{x}$ over $\mathcal{O}_{x}[H]$. Since $\ell_{0}$ is a generator of the generic stalk of $\mathcal{L}$ over $K[H]$ there exists for each $x \in X_{0}$ a unique element $\lambda_{x} \in K[H]^{*}$ such that $\ell_{x}=\lambda_{x} \ell_{0}$. Computing the stalk at a point $x^{\prime} \in X_{0}^{\prime}$ which lies over $x \in X_{0}$ one has

$$
\begin{aligned}
\mathcal{L}_{V^{*}, x^{\prime}}^{\prime} & =\bigoplus_{\kappa \mid V} H^{0}\left(H,\left(\mathcal{L}^{\prime} \otimes_{k} V_{\kappa^{*}}\right)_{x^{\prime}}\right) \\
& =\bigoplus_{\kappa \mid V} H^{0}\left(H,\left(\mathcal{L}_{x} \otimes_{k} k^{\prime}\right)_{x^{\prime}} \otimes_{k^{\prime}} V_{\kappa^{*}}\right)
\end{aligned}
$$




$$
\begin{aligned}
& =\bigoplus_{\kappa \mid V} e_{\kappa}\left(\mathcal{O}_{x}[H] \otimes_{k} k^{\prime}\right)_{x^{\prime}} \ell_{x} \\
& =\bigoplus_{\kappa \mid V} \kappa\left(\lambda_{x}\right) e_{\kappa}\left(\ell_{0}\right) \mathcal{O}_{x^{\prime}}^{\prime},
\end{aligned}
$$

and similarly

$$
\mathcal{F}_{V^{*}, x^{\prime}}^{\prime}=\bigoplus_{\kappa \mid V} e_{\kappa}\left(\ell_{0}\right) \mathcal{O}_{x^{\prime}}^{\prime}
$$

From (3.10-11) it follows that

$$
\begin{aligned}
\chi\left(\mathcal{F}_{V^{*}}\right) \Leftrightarrow \chi\left(\mathcal{L}_{V^{*}}\right) & =\Leftrightarrow \sum_{x^{\prime} \in X_{0}^{\prime}} \sum_{\kappa \mid V} \operatorname{deg}_{K(\phi)} \operatorname{Val}_{x^{\prime}}\left(\kappa\left(\lambda_{x}\right)\right) x^{\prime} \\
& =\Leftrightarrow \sum_{\kappa \mid V} \operatorname{deg}_{K(\phi)}\left(\sum_{x^{\prime} \in X_{0}^{\prime}} \operatorname{Val}_{x^{\prime}}\left(\kappa\left(\lambda_{x}\right)\right) x^{\prime}\right) \\
& =\Leftrightarrow \operatorname{dim}_{k}(V) \operatorname{deg}_{K(\phi)}\left(\theta_{\mathcal{O}[H]}(\mathcal{L}(\hat{\phi})),\right.
\end{aligned}
$$

with the last equality following form the $\Omega_{K}$-equivariance of $\operatorname{deg}_{K(\phi)}$, the explicit description of $\theta_{\mathcal{O}[H]}$ on the class of $\mathcal{L}$ (cf. Proposition 1.3), and the fact that $\hat{\phi}$ is a constituent of $V$. Putting together this expression with that of (3.8) and the definition (3.7) of the homomorphism $D_{X, H}$ one can now check directly that the square in Proposition 3.4 commutes.

Remark 3.5. Note that the map $D_{X, H}$ of (3.7) factors through the homomorphism

$$
\operatorname{Rag}_{H}: \operatorname{Hom}_{\Omega_{K}}\left(R_{H}, \operatorname{Pic}\left(K^{\prime}\right)\right) \rightarrow \operatorname{Hom}_{\Omega_{K}}\left(A_{\hat{H}}, \operatorname{Pic}\left(K^{\prime}\right)\right)
$$

as used in Theorem 2.9. Indeed, since $\operatorname{Hom}_{\Omega_{k}}\left(\Leftrightarrow, \operatorname{Pic}\left(K^{\prime}\right)\right)$ is contravariant left exact one has a natural identification of $\operatorname{ker}\left(\operatorname{Rag}_{H}\right)$ with $\operatorname{Hom}_{\Omega_{K}}\left(\hat{H}, \operatorname{Pic}\left(K^{\prime}\right)\right)$. The claim now follows since $\operatorname{Hom}_{\Omega_{K}}\left(\hat{H}, \operatorname{Pic}\left(K^{\prime}\right)\right)=\operatorname{Hom}_{\Omega_{K}}\left(\hat{H}, \operatorname{Pic}\left(K^{\prime}\right)_{\text {tors }}\right)=$ $\operatorname{Hom}_{\Omega_{K}}\left(\hat{H}, \operatorname{Pic}^{0}\left(K^{\prime}\right)\right)$.

The degrees of those divisor classes which occur in the descriptions of Theorem 2.9 are straightforward to compute and so via Lemma 3.1, Lemma 3.3 and Proposition 3.4 this result gives an explicit description of the realizable subsets $\tilde{\chi} R \Gamma_{G}\left(\Re_{X}^{0}(g)\right), \tilde{\chi} R \Gamma_{G}\left(\Re_{X}(G)\right)$ etc., of $\mathrm{Cl}(k[G])$. We shall for brevity leave the derivation of such explicit results to the reader. However, we note in passing that it may well be interesting to compare the results obtainable in this way with previous results of Ellingsrud and Lonsted [E,L] and Nakajama [N1], [N2] and [N3]. 
Let us now fix a finite field $k$ of characteristic $p$ and a finite abelian group $G$. It seems natural to define the "realizable subsets" $\Re_{k}^{0}(G)$ and $\Re_{k}(G)$ of $\mathrm{Cl}(k[G])$ by setting

$$
\Re_{k}^{0}(G):=\sum_{X} \operatorname{res}_{k[G]}^{k_{X}[G]} \tilde{\chi} R \Gamma_{G}\left(\Re_{X}^{0}(G)\right),
$$

and

$$
\Re_{k}(G):=\sum_{X} \operatorname{res}_{k[G]}^{k_{X}[G]} \tilde{\chi} R \Gamma_{G}\left(\Re_{X}(G)\right),
$$

where here the sums are taken over all smooth projective curves $X$ such that $k_{X} \supseteq k$, and $\operatorname{res}_{k[G]}^{k_{x}[G]}: \mathrm{Cl}\left(k_{x}[G]\right) \rightarrow \mathrm{Cl}(k[G])$ is the homomorphism induced by restriction of scalars. (This point of view is for example naturally suggested by the results in [Bu, Ch] and [Bu].) To give explicit "Hom-descriptions" of $\Re_{k}^{0}(G)$ and $\Re_{k}(G)$ by using the above results it is clearly sufficient for us to interpret restriction of scalars in terms of Hom-descriptions.

LEMMA 3.6. Let $k^{\prime}$ be a subfield of $k$. Let $F$ be a subfield of $\mathbb{Q}_{p}^{c}$ which contains $W(k)$, and let $F^{\prime}$ be any subfield of $F$ which contains $W\left(k^{\prime}\right)$. We let

$$
\mathcal{N}_{F / F^{\prime}}: \tilde{H}_{\Omega_{F}}\left(R_{H, p}, \mathbb{Q}_{p}^{c *}\right) \rightarrow \tilde{H}^{\prime o m} \Omega_{F^{\prime}}\left(R_{H, p}, \mathbb{Q}_{p}^{c *}\right)
$$

be the homomorphism defined by $\mathcal{N}_{F / F^{\prime}} \tilde{h}(\chi):=\prod_{\sigma \in \Omega_{F}^{\prime} / \Omega_{F}} \tilde{h}\left(\chi^{\sigma}\right)^{\sigma^{-1}}$. If $F / F^{\prime}$ is unramified, respectively totally ramified, and $\pi_{F^{\prime}}=\pi_{F}$, respectively $\pi_{F^{\prime}}=$ $\operatorname{Norm}_{F / F^{\prime}} \pi_{F}$, then the following square commutes:

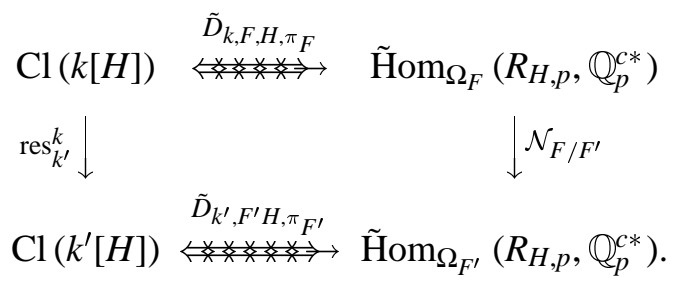

Proof. We write res for both of the functors $\operatorname{res}_{W\left(k^{\prime}\right)[H]}^{W(k)[H]}$ and $\operatorname{res}_{k^{\prime}[H]}^{k[H]}$. We let $T$ be a transversal to $\Omega_{F}$ and $\Omega_{F^{\prime}}$. Let $\bar{M}$ be a projective $k[H]$-module, with $M$ a projective $W(k)[H]$-module satisfying $M \otimes_{W(k)} k \cong \bar{M}$.

If $F / F^{\prime}$ is unramified then res $(M)$ is a projective $W\left(k^{\prime}\right)[H]$-module for which $\operatorname{res}(M) \otimes_{W\left(k^{\prime}\right)} k^{\prime} \cong \operatorname{res}(\bar{M})$. Now res $(M) \otimes_{W\left(k^{\prime}\right)} \mathbb{Q}_{p}^{c} \cong \oplus_{t \in T} M \otimes_{W(k), t} \mathbb{Q}_{p}^{c}$, and so for each $\chi \in R_{H, p}$ one has

$$
\begin{aligned}
\tilde{D}_{k^{\prime}, F^{\prime}, H, \pi_{F^{\prime}}} \circ \operatorname{res}(\bar{M})(\chi) & =\pi_{F^{\prime}}^{-\left\langle P \otimes_{W\left(k^{\prime}\right)} \mathbb{Q}_{p}^{c}, \chi\right\rangle} \\
& =\pi_{F}^{-\sum_{t \in T}\left\langle P \otimes_{W(k), t} \mathbb{Q}_{p}^{c}, \chi\right\rangle}
\end{aligned}
$$




$$
\begin{aligned}
& =\pi_{F}-\sum_{t \in T}\left\langle P \otimes_{W(k)} \mathbb{Q}_{p}^{c}, \chi^{t}\right\rangle \\
& =\mathcal{N}_{F / F^{\prime}} \circ D_{k, F, H, \pi_{F}}(\bar{M})(\chi),
\end{aligned}
$$

which is as is required.

We now assume that $F / F^{\prime}$ is totally ramified. In this case, if $M_{0}$ is a projective $W\left(k^{\prime}\right)[H]$-module which satisfies $M_{0} \otimes_{W\left(k^{\prime}\right)} k^{\prime} \cong \operatorname{res}(\bar{M})$ then $M_{0}^{\prime}:=M_{0} \otimes_{W\left(k^{\prime}\right)}$ $W(k)$ is a projective $W(k)[H]$-module which is such that $M_{0}^{\prime} \otimes_{W(k)} k \cong \bar{M}$. From Nakayama's lemma it follows that $M_{0}^{\prime}$ and $M$ are isomorphic $W(k)[H]$-modules. It follows in particular that $\chi_{P \otimes_{W(k)} \mathbb{Q}_{p}^{c}}=\chi_{P_{0} \otimes_{W\left(k^{\prime}\right)} \mathbb{Q}_{p}^{c}}$ and so this character is fixed under the action of $\Omega_{F^{\prime}}$. For each character $\psi \in R_{H, p}$ one therefore has

$$
\begin{aligned}
\mathcal{N}_{F / F^{\prime}} \circ D_{k, F, H, \pi_{F}}(\bar{M})(\psi) & =\prod_{t \in T}\left(\pi_{F}^{-\left\langle P \otimes_{W(k)} \mathbb{Q}_{p}^{c}, \psi^{t}\right\rangle}\right)^{t^{-1}} \\
& =\left(\operatorname{Norm}_{F / F^{\prime}}\left(\pi_{F}\right)\right)^{-\left\langle P \otimes_{W(k)} \mathbb{Q}_{p}^{c}, \psi\right\rangle} \\
& =D_{k^{\prime}, F^{\prime}, H, \pi_{F^{\prime}}}(\bar{M})(\psi),
\end{aligned}
$$

as is required.

Note that since any field extension $F / F^{\prime}$ contains an intermediate field $F_{0}$ such that $F / F_{0}$ is totally ramified and $F_{0} / F$ is unramified this last result is sufficient to deal with arbitrary restriction of scalars.

4. Characteristic zero realizable classes. In this section we shall outline how invertible sheaves on tame Galois $G$-covers of $X$ also naturally give rise to classes in Grothendieck groups of certain categories of modules over rings of characteristic 0 . Aside from any intrinsic interest such classes may have, they also arise in the context of obtaining explicit analogues of known Adams-RiemannRoch formulas. We shall however confine ourselves here to a brief discussion of this aspect of the theory, and defer a more detailed treatment to elsewhere.

For any Dedekind domain $R$ we let $K_{0} T(R[G])$ denote the Grothendieck group of the category of finite locally-freely-presented $R[G]$-modules. We let $A$ denote the ring of algebraic integers in a number field $K$, and for each $\wp \in \operatorname{Spec}(A)_{0}$ we let $k(\wp)$ denote the residue field of the completion of $A$ at $\wp$. There is a canonical isomorphism

$$
K_{0} T(A[G]) \cong \coprod_{\wp \in \operatorname{Spec}(A)_{0}} K_{0} T\left(A_{\wp}[G]\right)
$$

Furthermore, for each $\wp \in \operatorname{Spec}(A)_{0}$, and any choice of uniformizing parameter $\pi_{\wp}$ of $A_{\wp}$, the natural surjection

$$
\operatorname{Hom}_{\Omega_{K_{\wp}}}\left(R_{G, p}, \mathbb{Q}_{p}^{c *}\right) \rightarrow K_{0} T\left(A_{\wp}[G]\right)
$$


(cf. [F], II2.3) annihilates the subgroup generated by $D_{k(\wp), K_{\wp}, G, \pi_{\wp}}(k(\wp)[G])$ (cf. Remark 3.5). In conjunction with the injection $\tilde{D}_{k(\wp), K_{\wp}, G, \pi_{\wp}}$ of (3.6) the isomorphism (4.1) therefore gives a natural homomorphism

$$
\theta_{A, \wp, k, G}: \mathrm{Cl}(k[G]) \rightarrow K_{0} T(A[G])
$$

where here $\wp \in \operatorname{Spec}(A)_{0}$ and $k$ is finite field such that $W(k) \subseteq A_{\wp}$. We define the "geometrically realizable" subset of $K_{0} T(A[G])$ to be

$$
\Re T_{A}^{\text {geom }, 0}(G):=\sum_{\wp} \sum_{k} \theta_{A, \wp, k, G}\left(\Re_{k}^{0}(G)\right)
$$

and

$$
\Re T_{A}^{\text {geom }}(G):=\sum_{\wp} \sum_{k} \theta_{A, \wp, k, G}\left(\Re_{k}(G)\right)
$$

where here the sums are taken over all primes $\wp \in \operatorname{Spec}(A)_{0}$ and all finite fields $k$ such that $W(k) \subseteq A_{\wp}$. The images $\Re_{A}^{\text {geom,0 }}(G)$, respectively $\Re_{A}^{\text {geom }}(G)$ of $\Re T_{A}^{\text {geom }, 0}(G)$, respectively $\Re T_{A}^{\text {geom }}(G)$, under the natural surjection $K_{0} T(A[G]) \rightarrow \mathrm{Cl}(A[G])$ are then natural geometric counterparts to the subsets $\Re_{A, 0}(G)$, respectively $\Re_{A}(G)$, of "arithmetically realizable" classes of $\mathrm{Cl}(A[G])$ as considered in $[\mathrm{M}]$ and $[\mathrm{Bu}]$.

Examples 4.1. (i) A celebrated theorem of M. Taylor ([T]) implies that $\Re_{\mathbb{Z}, 0}(G)=\{0\}$, and a geometric analogue of Chinburg ([Ch2], Theorem 6.13) that $\Re_{\mathbb{Z}}^{\text {geom, } 0}(G)=\{0\}$.

(ii) If $G$ is an elementary abelian group of $l$-power order then one knows that $\Re_{\mathbb{Z}}(G)=\sum_{C \in \mathcal{C}}(G)$ ind $_{C}^{G} \mathrm{Cl}(\mathbb{Z}[C])$ (cf. [Bu], Theorem 1 and Remark 1.2(ii)). On the other hand, one can use the results of $\S 2$ and $\S 3$ to show that $\Re_{\mathbb{Z}}^{\text {geom }}(G) \subseteq$ $\sum_{C \in \mathcal{C}(G)} \operatorname{ind}_{C}^{G} \mathrm{Cl}(\mathbb{Z}[C])$ and is in addition annihilated by $l \Leftrightarrow 1$.

A more careful analysis of the material of $\S 2$ and $\S 3$ would allow one to describe explicitly the subsets $\Re T_{A}^{\text {geom }, 0}(G), \Re \mathfrak{T}_{A}^{\text {geom }}(G), \Re_{A}^{\text {geom }}(G)$ and $\Re_{A}^{\text {geom, } 0}(G)$ in terms of the appropriate Hom-descriptions. It may well be interesting to systematically compare results obtainable in this way with the results in $[\mathrm{M}]$ and [Bu] concerning the classes $\Re_{A, 0}(G)$ and $\Re_{A}(G)$. However, for brevity, we shall forego any such explicit analysis here.

We comment finally upon the connection between our results and certain types of Adams-Riemann-Roch theorems. An explicit analogue of the equivariant Adams-Riemann-Roch theorem is known for the subgroup of $K_{0} T(A[G])$ which is generated by classes arising from (differences of) $G$-stable invertible sheaves in tame Galois $G$-extensions of $K$ (cf. [Ch,E,P,T], Theorem 3.6). The techniques of $[\mathrm{Bu}, \mathrm{Ch}]$ on the other hand afford an explicit interpretation of the Cassou- 
Noguès-Taylor-Adams operators on the subgroups $\Re T_{A}^{\text {geom }}(G)$ of $K_{0} T(A[G])$ and $\Re_{A}(G)$ of $\mathrm{Cl}(A[G])$. Since explicit interpretations of the Cassou-Noguès-TaylorAdams operators on all elements of either $K_{0} T(A[G])$ or $\mathrm{Cl}(A[G])$ still seem to be difficult to obtain (see for example $[\mathrm{K}]$ ) it is of some interest to know just how big the subgroups $\Re_{k}(G), \Re T_{A}^{\text {geom }}(G), \Re_{A}(G)$, etc., can be.

5. Classes arising from structure sheaves. In this section we describe how the results of $[\mathrm{M}]$ may be adapted to give a proof of Theorem 2.9 (i) and (ii). We shall follow $[\mathrm{M}]$ very closely and so we refer to this paper for the details of most of the proofs, rather than repeating them word for word here.

We recall that $X$ is a smooth projective curve with constant field $k=k_{X}$, and function field $K=K_{X}$. Galois $K[H]$-algebras (or, alternatively, Galois $H$-covers of $X)$ are classified by continuous homomorphisms

$$
h: \Omega_{K} \rightarrow H .
$$

Corresponding to each such homomorphism $h$ we have a Galois $H$-extension $K_{h}$ of $K$ given by

$$
K_{h}:=\operatorname{Map}_{\Omega_{K}}\left({ }^{h} H, K^{c}\right)
$$

where here ${ }^{h} H$ is the left $\Omega_{K}$-set consisting of the set $H$ with $\Omega_{K}$ acting by left multiplication via $h$, and $K_{h}$ is the algebra of $K^{c}$-valued functions on $H$ which preserve the action of $\Omega_{K}$. So, for $a \in K_{h}$, we have $a(h(\omega) s)=a(s)^{\omega}$ for each $s \in H$ and $\omega \in \Omega$. It is clear that the Galois $H$-extensions $K_{h}$ of $K$ all lie in the $K^{c}$-algebra $\operatorname{Map}\left(H, K^{c}\right)$.

Set $K^{h}=\left(K^{c}\right)^{\operatorname{ker}(h)}$, the fixed field of $\operatorname{ker}(h)$. Then $K_{h}$ is isomorphic as a $K$-algebra to a product of $\left[H: h\left(\Omega_{K}\right)\right]$ copies of $K^{h}$; this isomorphism depends upon a choice of coset representatives for $h\left(\Omega_{K}\right) \backslash H$.

We shall now describe the "resolvend map"

$$
\tilde{r}_{H}: \operatorname{Map}\left(H, K^{c}\right) \rightarrow K^{c}[H] .
$$

This is defined by associating to each element $a \in \operatorname{Map}\left(H, K^{c}\right)$ its "resolvend"

$$
\tilde{r}_{H}(a):=\sum_{s \in H} a(s) s^{-1} .
$$

This map $\tilde{r}_{H}$ is an isomorphism of $K^{c}$-vector spaces (but not of algebras since it does not preserve multiplication). For $a \in \operatorname{Map}\left(H, K^{c}\right)$ we have

$$
a \in K_{h} \Leftrightarrow \tilde{r}_{H}(a)^{\omega}=\tilde{r}_{H}(a) h(\omega) \text { for all } \omega \in \Omega_{K} .
$$

(Here $\Omega_{K}$ acts on $K^{c}[H]$ via its natural action on the coefficients.) 
We say that an element $a \in K_{h}$ generates a normal basis of $K_{h} / K$ if $K_{h}=$ $K[H] a$. The use of resolvends enables one to give the following criterion for $a \in K_{h}$ to be a normal basis generator of $K_{h} / K$.

Proposition 5.1. For $a \in K_{h}$ we have

$$
K_{h}=K[H] a \Leftrightarrow \tilde{r}_{H}(a) \in\left(K^{c}[H]\right)^{*} .
$$

Proof. Exactly as in Proposition 1.8 of $[\mathrm{M}]$.

We shall now consider the group $\operatorname{Hom}\left(\Omega_{K}, H\right)$. We begin by considering the following exact sequence of $\Omega_{K}$-modules (here $\Omega_{K}$ acts trivially on $H$ )

$$
1 \rightarrow H \rightarrow\left(K^{c}[H]\right)^{*} \rightarrow\left(K^{c}[H]\right)^{*} / H \rightarrow 1 .
$$

It may be shown that taking $\Omega_{K^{-}}$-cohomology of (5.1) yields the exact sequence

$$
1 \rightarrow H \rightarrow(K[H])^{*} \rightarrow \mathcal{H}(K[H]) \rightarrow \operatorname{Hom}\left(\Omega_{K}, H\right) \rightarrow 1
$$

where here $\mathcal{H}(K[H])=\left(\left(K^{c}[H]\right)^{*} / H\right)^{\Omega_{K}}$ (cf. [M], 1.19).

Setting

$$
\mathfrak{H}(K[H]):=\left\{\alpha \in\left(K^{c}[H]\right)^{*} \mid \alpha^{\omega-1} \in H \quad \text { for all } \omega \in \Omega_{K}\right\}
$$

we have $\mathcal{H}(K[H])=\mathfrak{H}(K[H]) / H$. Note also that

$$
\mathfrak{H}(K[H])=\left\{\tilde{r}_{H}(a) \mid K[H] a=K_{h} \quad \text { for some } \quad h \in \operatorname{Hom}\left(\Omega_{K}, H\right)\right\} .
$$

We recall (from the proof of Proposition 1.3) that there is a natural identification $(K[H])^{*}=\operatorname{Hom}_{\Omega_{K}}\left(\mathbb{Z}[\hat{H}],\left(K^{c}\right)^{*}\right)$.

Now consider the exact sequence

$$
0 \rightarrow A_{\hat{H}} \rightarrow \mathbb{Z}[\hat{H}] \stackrel{\text { det }}{\rightarrow} \hat{H} \rightarrow 1
$$

where here det: $\mathbb{Z}[\hat{H}] \rightarrow \hat{H}$ is defined by

$$
\operatorname{det}\left(\sum_{\chi \in \hat{H}} a_{\chi} \chi\right)=\prod_{\chi \in \hat{H}} \chi^{a_{\chi}} \quad\left(a_{\chi} \in \mathbb{Z}\right),
$$

and $A_{\hat{H}}$ denotes the kernel of det. Applying the functor $\operatorname{Hom}\left(\Leftrightarrow,\left(K^{c}\right)^{*}\right)$ to (5.3) yields the exact sequence

(5.4) $1 \rightarrow \operatorname{Hom}\left(\hat{H},\left(K^{c}\right)^{*}\right) \rightarrow \operatorname{Hom}\left(\mathbb{Z}[\hat{H}],\left(K^{c}\right)^{*}\right) \rightarrow \operatorname{Hom}\left(A_{\hat{H}},\left(K^{c}\right)^{*}\right) \rightarrow 1$. 
We identify the terms of the sequence (5.4) with the corresponding terms of the sequence (5.1) as follows:

$$
\left\{\begin{aligned}
\operatorname{Hom}\left(\hat{H},\left(K^{c}\right)^{*}\right) & \leftrightarrow H \\
\operatorname{Hom}\left(\mathbb{Z}[\hat{H}],\left(K^{c}\right)^{*}\right) & \leftrightarrow\left(K^{c}[H]\right)^{*} \\
\operatorname{Hom}\left(A_{\hat{H}},\left(K^{c}\right)^{*}\right) & \leftrightarrow\left(K^{c}[H]\right)^{*} / H
\end{aligned}\right\}
$$

These identifications allow us to give an alternative description of the middle portion $(K[H])^{*} \rightarrow \mathcal{H}(K[H])$ for the sequence (5.2): we have

$$
\operatorname{Hom}_{\Omega_{K}}\left(\mathbb{Z}[\hat{H}],\left(K^{c}\right)^{*}\right) \underset{\operatorname{rag}_{H}}{\longrightarrow} \operatorname{Hom}_{\Omega_{K}}\left(A_{\hat{H}},\left(K^{c}\right)^{*}\right)=\operatorname{Hom}_{\Omega_{K}}\left(A_{\hat{H}},\left(K^{\prime}\right)^{*}\right),
$$

where here the map $\operatorname{rag}_{H}$ denotes restriction to $A_{\hat{H}}$ (that is, restriction to augmentation).

Suppose that $a \in K_{h}$ generates a normal basis of $K_{h} / K$. Its resolvend $\tilde{r}_{H}(a)$ is a homomorphism from $\mathbb{Z}[\hat{H}]$ to $\left(K^{c}\right)^{*}$. We let $r_{H}(a): A_{\hat{H}} \rightarrow\left(K^{c}\right)^{*}$ denote the restriction of $\tilde{r}_{H}(a)$ to $A_{\hat{H}}$, and we call $r_{H}(a)$ the reduced resolvend of $a$. Thus, $r_{H}(a)$ may be identified with the coset of $\tilde{r}_{H}(a)$ in $\left(K^{c}[H]\right)^{*} / H$, and we have

$$
\mathcal{H}(K[H])=\left\{r_{H}(a) \mid K[H] a=K_{h} \quad \text { for some } h \in \operatorname{Hom}\left(\Omega_{K}, H\right)\right\}
$$

We shall now discuss tame $H$-extensions of $K$. For each closed point $x \in X_{0}$ we choose and fix an algebraic closure $K_{x}^{c}$ of the completion $K_{x}$ of $K$ at $x$, and an embedding $i_{x}: K^{c} \rightarrow K_{x}^{c}$ of $K^{c}$ into $K_{x}^{c}$, and we let $\tilde{i}_{x}: \Omega_{K_{x}} \rightarrow \Omega_{K}$ denote the corresponding embedding of absolute Galois groups. (We shall regard $i_{x}$ and $\tilde{i}_{x}$ as inclusions and so shall suppress them from the notation when there is no danger of confusion.)

If $h \in \operatorname{Hom}\left(\Omega_{K}, H\right)$ we set $h_{x}:=h \circ i_{x} \in \operatorname{Hom}\left(\Omega_{K}, H\right)$. Then

$$
\left(K_{x}\right)_{h_{x}}=K_{h} \otimes_{K} K_{x}
$$

and we have $K_{h} \subseteq\left(K_{x}\right)_{h_{x}}$. We let $\mathcal{O}_{h_{x}}$, respectively $\mathcal{O}_{x}^{c}$, denote the integral closure of $\mathcal{O}_{x}$ in $\left(K_{x}\right)_{h_{x}}$, respectively $K_{x}^{c}$.

We write $\Omega_{K}^{t}$, respectively $\Omega_{K_{x}}^{t}$, for the Galois group of the maximal tame extension $K^{t} / K$, respectively $K_{x}^{t} / K_{x}$, of $K$, respectively $K_{x}$. Then $\tilde{i}_{x}$ restricts to $\tilde{i}_{x}: \Omega_{K_{x}}^{t} \rightarrow \Omega_{K}^{t}$, and a Galois $H$-extension $K_{h} / K$ is tame if and only if the homomorphism $h: \Omega_{K} \rightarrow H$ factors through the quotient map $\Omega_{K} \rightarrow \Omega_{K}^{t}$, or equivalently, if and only if $K^{h} \subseteq K^{t}$. So, if we regard $\operatorname{Hom}\left(\Omega_{K}^{t}, H\right)$ as a subset of $\operatorname{Hom}\left(\Omega_{K}, H\right)$ then $K_{h} / K$ is tame if and only if $h \in \operatorname{Hom}\left(\Omega_{K}^{t}, H\right)$. In this case, we shall also say that $h$ "is tame" (a similar discussion holds with $K$ replaced by $\left.K_{x}\right)$. Hence, $h$ is tame if and only if $h_{x}$ is tame for all $x \in X_{0}$.

We let $\left.\mathcal{H}^{t}(K[H])\left(:=\mathfrak{H}^{t}(K[H]) / H\right)\right)$ denote the pre-image of $\operatorname{Hom}\left(\Omega_{K}^{t}, H\right)$ under the connecting homomorphism $c: \mathcal{H}(K[H]) \rightarrow \operatorname{Hom}\left(\Omega_{K}, H\right)$ (with similar 
notation for $K_{x}$ ). Similarly, we let $\Omega_{x}^{n r}$ denote the Galois group of the maximal unramified extension $K_{x}^{n r} / K_{x}$ in $K_{x}^{c}$, and we write $\mathcal{H}^{n r}\left(K_{x}[H]\right)\left(:=\mathfrak{H}^{n r}\left(K_{x}[H]\right) / H\right)$ for the pre-image of $\operatorname{Hom}\left(\Omega_{x}^{n r}, H\right)$ under $c$.

We shall now describe integral versions of (5.1) and the constructions that arise therefrom. For each $x \in X_{0}$ we consider the exact sequence

$$
1 \rightarrow H \rightarrow\left(\mathcal{O}_{x}^{c}[H]\right)^{*} \rightarrow\left(\mathcal{O}_{x}^{c}[H]\right)^{*} / H \rightarrow 1
$$

It may be shown that taking $\Omega_{K_{x}}$-cohomology of this sequence yields an exact sequence

$$
1 \rightarrow H \rightarrow\left(\mathcal{O}_{x}[H]\right)^{*} \rightarrow \mathcal{H}\left(\mathcal{O}_{x}[H]\right) \rightarrow \operatorname{Hom}\left(\Omega_{K_{x}}^{n r}, H\right) \rightarrow 1
$$

(cf. [M], 2.12). Here

$$
\mathcal{H}\left(\mathcal{O}_{x}[H]\right)=\left(\left(\mathcal{O}_{x}^{c}[H]\right)^{*} / H\right)^{\Omega_{K}}=\mathfrak{H}\left(\mathcal{O}_{x}[H]\right) / H
$$

where

$$
\mathfrak{H}\left(\mathcal{O}_{x}[H]\right)=\left(\mathcal{O}_{x}^{c}[H]\right)^{*} \cap \mathfrak{H}\left(K_{x}[H]\right)
$$

Hence we have

$$
\mathcal{H}\left(\mathcal{O}_{x}[H]\right)=\left\{r_{H}\left(a_{x}\right) \mid \mathcal{O}_{x}[H] . a_{x}=\left(\mathcal{O}_{x}\right)_{h_{x}} \quad \text { for some } \quad h_{x} \in \operatorname{Hom}\left(\Omega_{K_{x}}^{n r}, H\right)\right\}
$$

and

$$
\mathcal{H}^{n r}\left(K_{x}[H]\right)=\mathcal{H}\left(\mathcal{O}_{x}[H]\right) \cdot \operatorname{rag}_{H}\left(\left(K_{x}[H]\right)^{*}\right)
$$

or equivalently

$$
\mathfrak{H}^{n r}\left(K_{x}[H]\right)=\mathfrak{H}\left(\mathcal{O}_{x}[H]\right) .\left(K_{x}[H]\right)^{*} .
$$

We also observe that since the characteristic of $k$ does not divide \#H it follows from (5.6) and (5.5) that

$$
\mathcal{H}\left(\mathcal{O}_{x}[H]\right)=\operatorname{Hom}\left(A_{\hat{H}},\left(\mathcal{O}_{x}^{c}\right)^{*}\right)^{\Omega_{K}} \quad \text { for all } \quad x \in X_{0}
$$

(cf. [M], Theorem 2.14).

We now take an $H$-cover $f: Y \rightarrow X$ of $X$, and we let $h: \Omega_{K} \rightarrow H$ be the corresponding continuous homomorphism. Set $\mathcal{O}_{h}:=f_{*} \mathcal{O}_{Y}$; then $\mathcal{O}_{h}$ is a rank one locally free $\mathcal{O}[H]$-module. Let $\eta$ denote the generic point of $X$; then we have that $\left(\mathcal{O}_{h}\right)_{\eta} \cong K_{h}$. For each $x \in X$ the stalk $\left(\mathcal{O}_{h}\right)_{x}$ is freely generated over $\mathcal{O}_{x}[H]$ by an element $a_{x}$, say. (We refer to $a_{x}$ as a normal integral basis of $\left(\mathcal{O}_{h}\right)_{x}$ over $\mathcal{O}_{x}$.) Furthermore, each $\left(\mathcal{O}_{h}\right)_{x}$ may be regarded as an $\mathcal{O}_{x}[H]$-lattice in the free 
$K_{h}$-module $K_{h}$ (cf. the discussion immediately preceding Proposition 1.3), and so $a_{x}=c_{x} a_{\eta}$ for a unique $c_{x} \in\left(K_{x}[H]\right)^{*}$. Then $\left(c_{x}\right)_{x \in X_{0}} \in J_{K}(K[H])$ and it follows from the proof of Proposition 1.3 that $\left(c_{x}\right)_{x \in X_{0}}$ is representative of the class of the $\mathcal{O}[H]$-module $\mathcal{O}_{h}$ in $\operatorname{Pic}(\mathcal{O}[H])$.

The equation $a_{x}=c_{x} a_{\eta}$ may be expressed in terms of resolvends. We have

$$
\tilde{r}_{h}\left(a_{x}\right)=c_{x} \cdot \tilde{r}_{H}\left(a_{\eta}\right)
$$

in $\mathfrak{H}\left(K_{x}[H]\right)$, and this implies that

$$
r_{H}\left(a_{x}\right)=\operatorname{rag}_{H}\left(c_{x}\right) r_{H}\left(a_{\eta}\right)
$$

in $\mathcal{H}\left(K_{x}[H]\right)$, that is

$$
\operatorname{rag}_{H}\left(c_{x}\right)=r_{H}\left(a_{\eta}\right) r_{H}\left(a_{x}\right)^{-1}
$$

Next, we observe that if $\operatorname{rag}_{H}\left(c_{x}^{\prime}\right)=\operatorname{rag}_{H}\left(c_{x}\right)$ for all $x \in X$ then $c_{x}^{\prime}=c_{x} s_{x}$ with $s_{x} \in H$ for all $x$. Thus $\left(s_{x}\right)_{x} \in \prod_{x \in X}\left(\mathcal{O}_{x}[H]\right)^{*}$ and so $\left(c_{x}^{\prime}\right)_{x}$ determines the same class in $\operatorname{Pic}(\mathcal{O}[H])$ as does $\left(c_{x}\right)_{x}$. Hence in fact $\left(\operatorname{rag}_{H}\left(c_{x}\right)\right)_{x}$ determines the class of $\mathcal{O}_{h}$ in $\operatorname{Pic}(\mathcal{O}[H])$.

Now $\operatorname{rag}_{H}\left(c_{x}\right)$ is not arbitrary, but has in fact a nice local decomposition. This decomposition involves the Stickelberger map which we shall now define.

We fix a root of unity $\mu$ in $k^{c}$ of order equal to the exponent of $H$. For $\chi \in \hat{H}$ and $s \in H$ we let $\langle\chi, s\rangle_{\mu}$ denote the unique rational number lying between 0 and 1 for which $\chi(s)=\mu^{\exp (H) \cdot\langle\chi, s\rangle_{\mu}}$, and we extend this to give a $\mathbb{Q}$-bilinear map

$$
\langle,\rangle_{\mu}: \mathbb{Q}[\hat{H}] \times \mathbb{Q}[H] \rightarrow \mathbb{Q}
$$

The Stickelberger map (relative to $\mu$ )

$$
\Theta_{H, \mu}: \mathbb{Q}[\hat{H}] \rightarrow \mathbb{Q}[H]
$$

is then defined by

$$
\Theta_{H, \mu}(\alpha)=\sum_{s \in H}\langle\alpha, s\rangle_{\mu} s, \quad \text { for each } \quad \alpha \in \mathbb{Q}[\hat{H}] .
$$

For any $\omega \in \Omega_{k}$ on has

$$
\Theta_{H, \mu^{\omega}}=\Theta_{H, \mu} \circ \omega^{-1} .
$$

The Stickelberger module is then defined to be

$$
S_{h}:=\Theta_{H, \mu}(\mathbb{Z}[\hat{H}]) \cap \mathbb{Z}[H],
$$


and is independent of the choice of $\mu$ as a consequence of (5.8). We define $H(\Leftrightarrow 1)$ to be the group $H$ endowed with an $\Omega_{K}$-module structure via the inverse cyclotomic character.

The following result is proved exactly as in [M] (see Propositions 4.3 and 4.5 (ibid.)), and so we shall not repeat the proof here.

Proposition 5.2. (i) For each $\alpha \in \mathbb{Z}[\hat{H}]$ we have $\Theta_{H, \mu}(\alpha) \in \mathbb{Z}[H]$ if and only if $\alpha \in A_{\hat{H}}$. In particular therefore, $\Theta_{H, \mu}$ defines by restriction a homomorphism $\Theta_{H, \mu}: A_{\hat{H}} \rightarrow \mathbb{Z}[H]$ and one has $S_{H}=\Theta_{H, \mu}\left(A_{\hat{H}}\right)$.

(ii) The linear transformation $\Theta_{H, \mu}: \mathbb{Q}[\hat{H}] \rightarrow \mathbb{Q}[H](\Leftrightarrow 1)$ preserves the action of $\Omega_{K}$.

Proposition 5.2 implies that the transpose map

$$
\Theta_{H, \mu}^{t}: \operatorname{Hom}\left(\mathbb{Z}[H](\Leftrightarrow 1),\left(K^{c}\right)^{*}\right) \rightarrow \operatorname{Hom}\left(A_{\hat{H}},\left(K^{c}\right)^{*}\right)
$$

is an $\Omega_{K}$-homomorphism, where $\Omega_{K}$ acts on homomorphisms by $f^{\omega}(\chi)=f\left(\chi^{\omega^{-1}}\right)^{\omega}$ (for each $\omega \in \Omega_{K}$ ), and $\Theta_{H, \mu}^{t}(f)=f \circ \Theta_{H, \mu}$. Hence we also have the homomorphism (which we also denote by $\Theta_{H, \mu}^{t}$ ) given by

$$
\Theta_{H, \mu}^{t}: \operatorname{Hom}_{\Omega_{K}}\left(\mathbb{Z}[H](\Leftrightarrow 1),\left(K^{c}\right)^{*}\right) \rightarrow \operatorname{Hom}_{\Omega_{K}}\left(A_{\hat{H}}\left(K^{c}\right)^{*}\right)=\mathcal{H}(K[H]) .
$$

For each $\Omega_{K}$-orbit $t$ of $H(\Leftrightarrow 1)$ we let $K(t)$ denote the constant field extension of $K$ which corresponds by Galois theory to the pointwise stabilizer of $t$ in $\Omega_{K}$. Then we have an isomorphism

$$
\operatorname{Map}_{\Omega_{K}}\left(H(\Leftrightarrow 1), K^{c}\right) \cong \prod_{t \in H(-1) / \Omega_{K}} K(t) .
$$

For each $x \in X_{0}$ we also let $\Lambda_{x}$ denote $\operatorname{Map}_{\Omega_{K x}}\left(H(\Leftrightarrow 1), \mathcal{O}_{x}^{c}\right)$, and we let $K \Lambda$ denote $\operatorname{Map}_{\Omega_{K}}\left(H(\Leftrightarrow 1), K^{c}\right)$. Then the transpose $\Theta_{H, \mu}^{t}$ may be expressed as a map $\Theta_{H, \mu}^{t}:(K \Lambda)^{*} \rightarrow \mathcal{H}(K[H])$.

If for each $x \in X_{0}$ we let $K_{x} \Lambda$ denote $\operatorname{Map}_{\Omega_{K x}}\left(H(\Leftrightarrow 1), K_{x}^{c}\right)$ then there are corresponding local homomorphisms $\Theta_{H, \mu}^{t}:\left(K_{x} \Lambda\right)^{*} \rightarrow \mathcal{H}\left(K_{x}[H]\right)$.

We now turn to describe the natural decomposition of the resolvends of local normal integral basis generators which we referred to above. For each $x \in X_{0}$ this involves the determination of a distinguished set of coset representatives for $\mathcal{H}^{t}\left(K_{x}[H]\right) / \mathcal{H}^{n r}\left(K_{x}[H]\right)$ of the form $\Theta_{H, \mu}^{t}\left(f_{x}\right)$ for suitable elements $f_{x}$ of $\left(K_{x} \Lambda\right)^{*}$.

We shall for the moment fix a point $x \in X_{0}$. We let $\pi\left(=\pi_{x}\right)$ denote a fixed generator of the maximal ideal $\mathfrak{m}_{x}$ of $\mathcal{O}_{x}$, and we let $q_{x}$ denote the order of the residue field $\mathcal{O}_{x} / \mathfrak{m}_{x}$. The field $K_{x}^{n r}$ is obtained by adjoining all roots of unity to $K_{x}$. The group $\Omega_{x}^{n r}:=\operatorname{Gal}\left(K_{x}^{n r} / K_{x}\right)$ is procyclic and is generated by the Frobenius automorphism $\phi\left(=\phi_{x}\right)$ of $K_{x}^{n r} / K_{x}$ which is given by $\phi(\xi)=\xi^{q_{x}}$ for each root of unity $\xi$. The field $K_{x}^{t}$ is obtained by adjoining to $K_{x}^{n r}$ the values $\pi^{1 / n}$ for all 
integers $n$ coprime to $p$. The group $\operatorname{Gal}\left(K_{x}^{t} / K_{x}^{n r}\right)$ is also procyclic, and we fix a generator $\sigma$ of it. We fix a coherent set of radicals $\left\{\pi^{1 / n}\right\}$ for all integers $n$ coprime to $p$ such that $\left(\pi^{1 / m n}\right)^{n}=\pi^{1 / m}$. Then this gives a collection $\left\{\mu_{n}\right\}$ of roots of unity defined by

$$
\sigma\left(\pi^{1 / n}\right)=\mu_{n} \pi^{1 / n}
$$

We let $\phi$ also denote the unique lifting of $\pi$ from $\Omega_{x}^{n r}$ to $\Omega_{x}^{t}$ which fixes the distinguished elements $\pi^{1 / n}$ for each $n$ coprime to $p$. Then $\Omega_{x}^{t}$ is generated by $\sigma$ and $\phi$ and we have $\phi \circ \sigma \circ \phi^{-1}=\phi^{q}$. (This last equality can be seen by comparing the effects of both sides on the elements $\pi^{1 / n}$ and $\mu_{n}$.) The abeliniazation $\left(\Omega_{x}^{t}\right)^{a b}$ of $\Omega_{x}^{t}$ is given by

$$
\left(\Omega_{x}^{t}\right)^{a b}=\langle\bar{\sigma}\rangle \times\langle\bar{\phi}\rangle
$$

where here we write $\bar{\sigma}$ and $\bar{\phi}$ for the obvious images of $\sigma$ and $\phi$. Note that $\bar{\sigma}$ is of order $q \Leftrightarrow 1$ whilst $\langle\bar{\phi}\rangle$ is procyclic. We remark that since $H$ is abelian we have

$$
\operatorname{Hom}\left(\Omega_{x}^{t}, H\right)=\operatorname{Hom}\left(\left(\Omega_{x}^{t}\right)^{a b}, H\right)
$$

and this is why we are interested in the group $\left(\Omega_{x}^{t}\right)^{a b}$.

There is a natural homomorphism $h \mapsto h(\sigma)$ from $\operatorname{Hom}\left(\Omega_{x}^{t}, H\right)$ to $H$ which is given by "evaluating at $\sigma$. ." The kernel of this homomorphism is $\operatorname{Hom}\left(\Omega_{x}^{n r}, H\right)$. We let $H_{(q-1)}$ denote the subgroup of $H$ consisting of elements of order dividing $q \Leftrightarrow 1$. Since $h(\sigma)$ can take any value in $H_{(q-1)}$ we have the following sequence of isomorphisms

$$
\mathcal{H}^{t}\left(K_{x}[H]\right) / \mathcal{H}^{n r}\left(K_{x}[H]\right) \cong \operatorname{Hom}\left(\Omega_{x}^{t}, H\right) / \operatorname{Hom}\left(\Omega_{x}^{n r}, H\right) \cong H_{(q-1)} .
$$

We shall now describe a section of the induced homomorphism $\mathcal{H}^{t}\left(K_{x}[H]\right) \rightarrow$ $H_{(q-1)}$; this section depends upon the choice of distinguished prime element $\pi_{x}$. For each $s \in H_{(q-1)}$ we define

$$
f_{x, s} \in\left(K_{x} \Lambda\right)^{*}=\operatorname{Hom}_{\Omega_{K}}\left(\mathbb{Z}[H(\Leftrightarrow 1)],\left(K_{x}^{c}\right)^{*}\right)
$$

by setting (for each $t \in H$ )

$$
f_{x, s}(t):= \begin{cases}\pi_{x}, & \text { if } t=s \neq 1 \\ 1, & \text { otherwise. }\end{cases}
$$

Proposition 5.3. Suppose that $s \in H_{(q-1)}$ has ordere, and let $h_{x} \in \operatorname{Hom}\left(\Omega_{x}^{t}, H\right)$ be defined by $h_{x}\left(\sigma_{x}\right)=s$ and $h\left(\phi_{x}\right)=1$. The $K_{x}^{h_{x}}=K_{x}\left(\pi_{x}^{1 / e}\right)$ and $\Theta_{H, \mu}^{t}\left(f_{x, s}\right)=r_{H}\left(b_{x}\right)$ where $b_{x}$ generates a normal integral basis of $\left(\mathcal{O}_{x}\right)_{h_{x}}$ over $\mathcal{O}_{x}$. In particular we have 
$\Theta_{H, \mu}^{t}\left(f_{x, s}\right) \in \mathcal{H}^{t}\left(K_{x}[H]\right)$, and $\Theta_{H, \mu}^{t}\left(f_{x, s}\right)$ is sent to $s$ under the map $\mathcal{H}^{t}\left(K_{x}[H]\right) \rightarrow$ $H_{(q-1)}$.

Proof. The proof of this result is identical to that of Proposition 5.4 of [M].

As section to the homomorphism $\mathcal{H}^{t}\left(K_{x}[H]\right) \rightarrow H_{(q-1)}$ we shall take the map $s \mapsto \Theta_{H, \mu}^{t}\left(f_{x, s}\right)$.

The local decomposition theorem below reflects the fact that any tame extension of $K_{x}$ with ramification index $e$ is contained in the compositum of $K_{x}\left(\pi^{1 / e}\right)$ and an unramified extension. It is proved in exactly the same manner as Theorem 5.6 of $[\mathrm{M}]$.

THEOREM 5.4. (Local decomposition theorem) Suppose that $h_{x} \in \operatorname{Hom}\left(\Omega_{x}^{t}, H\right)$. If $a_{x}$ is a normal integral basis generator of $\left(\mathcal{O}_{x}\right)_{h_{x}}$ over $\mathcal{O}_{x}$ then we have

$$
r_{H}\left(a_{x}\right)=\Theta_{H, \mu}^{t}\left(f_{x, s}\right) u_{x}
$$

where here $h_{x}\left(\sigma_{x}\right)=s \in H_{(q-1)}$ and $u_{x} \in \mathcal{H}\left(\mathcal{O}_{x}[H]\right)$.

Conversely, let $s \in H_{(q-1)}$ and $u_{x} \in \mathcal{H}\left(\mathcal{O}_{x}[H]\right)$, and let $h_{x}$ be the image of $\Theta_{H, \mu}^{t}\left(f_{x, s}\right) u_{x}$ under the connecting homomorphism $\mathcal{H}^{t}\left(K_{x}[H]\right) \rightarrow \operatorname{Hom}\left(\Omega_{x}^{t}, H\right)$. Then $h_{x}\left(\sigma_{x}\right)=s$, and $\left(\mathcal{O}_{x}\right)_{h_{x}}$ has a normal integral basis generator $a_{x}$ over $\mathcal{O}_{x}$ for which $r_{H}\left(a_{x}\right)=\Theta_{H, \mu}^{t}\left(f_{x, s}\right) u_{x}$.

We can now turn to consider realizable classes.

We let $J_{K}(K \Lambda)$ denote the restricted direct product of the $\left(K_{x} \Lambda\right)^{*}$ with respect to the subgroups $\Lambda_{x}^{*}$ for $x \in X_{0}$. We let $\mathcal{H}(\mathbb{A}(K[G])), \mathcal{H}^{t}(\mathbb{A}(K[G]))$ and $\mathcal{H}^{n r}(\mathbb{A}(K[G]))$ respectively be the restricted direct products of the groups $\mathcal{H}\left(K_{x}[H]\right)$, $\mathcal{H}^{t}\left(K_{x}[H]\right)$ and $\mathcal{H}^{n r}\left(K_{x}[H]\right)$ with respect to the subgroups $\mathcal{H}\left(\mathcal{O}_{x}[H]\right)$. Then we may define maps

$$
\begin{gathered}
\Theta_{H, \mu}^{t}: J_{K}(K \Lambda) \rightarrow \mathcal{H}(\mathbb{A}(K[H])) \\
\operatorname{rag}_{H}: J_{K}(K[H]) \rightarrow \mathcal{H}(\mathbb{A}(K[H]))
\end{gathered}
$$

componentwise in the obvious manner. It is easy to check that these maps are well defined. Furthermore, since the characteristic $p$ of $K$ does not divide \#H we have (cf. (5.7))

$$
\Theta_{H, \mu}^{t}\left(\Lambda_{x}^{*}\right) \subseteq \operatorname{Hom}_{\Omega_{K}}\left(A_{\hat{H}},\left(\mathcal{O}_{x}^{c}\right)^{*}\right)=\mathcal{H}\left(\mathcal{O}_{x}[H]\right)
$$

We define the unit idele groups

$$
U(\Lambda):=\prod_{x \in X_{0}}\left(\Lambda_{x}\right)^{*}, \quad U(\mathcal{O}[H]):=\prod_{x \in X_{0}}\left(\mathcal{O}_{x}[H]\right)^{*}, \quad \mathcal{H}(\mathbb{A}(\mathcal{O}[H])):=\prod_{x \in X_{0}} \mathcal{H}\left(\mathcal{O}_{x}[H]\right)
$$


so that both

$$
\Theta_{H, \mu}^{t}(U(\Lambda)) \subseteq \mathcal{H}(\mathbb{A}(\mathcal{O}[H])), \quad \text { and } \quad \operatorname{rag}_{H}(U(\mathcal{O}[H])) \subseteq \mathcal{H}(\mathbb{A}(\mathcal{O}[H]))
$$

We let

$$
\lambda:(K \Lambda)^{*} \rightarrow J_{K}(K \Lambda), \quad \lambda: \mathcal{H}(K[H]) \rightarrow \mathcal{H}(\mathbb{A}(K[H]))
$$

denote the principal idele maps given by the natural diagonal embeddings. Then we have

$$
\lambda\left(\mathcal{H}^{t}(K[H])\right)=\lambda(\mathcal{H}(K[H])) \cap \mathcal{H}^{t}(\mathbb{A}(K[H])),
$$

and

$$
\lambda\left(\mathcal{H}^{n r}(K[H])\right)=\lambda(\mathcal{H}(K[H])) \cap \mathcal{H}^{n r}(\mathbb{A}(K[H])) .
$$

For each $x \in X_{0}$ we let $F_{x}\left(\subseteq\left(K_{x} \Lambda\right)^{*}\right)$ be the set of all $f_{x, s}$ for $s \in H_{(q-1)}$. As $q \Leftrightarrow 1$ is the order of the group of roots of unity in $K_{x}$ we have that $s \in H_{(q-1)}$ if and only if $K_{x}(s)=K_{x}$, and so $F_{x}=\left\{f_{x, s} \mid K_{x}(s)=K_{x}\right\}$.

We let $F_{X, H} \subset J_{K}(K \Lambda)$ be the subset of ideles $f$ for which $f_{x} \in F_{x}$ for all $x \in X_{0}$. It is easy to see that if $f \in F_{X, H}$ then $f_{x}=1$ for all but finitely many $x \in X_{0}$. We refer to the nontrivial elements of $F_{X} \subset F_{X, H}$ as the "prime $F$-elements" lying over $x$. It follows that elements of $F_{X, H}$ are finite products of prime $F$-elements lying over distinct $x \in X_{0}$. From Proposition 5.3 we have $\Theta_{H, \mu}^{t}(f) \in \mathcal{H}^{t}(\mathbb{A}(K[H]))$ for all $f \in F_{X, H}$.

We let

$$
\rho: J_{K}(K[H]) \rightarrow \frac{J_{K}(K[H])}{(K[H])^{*} U(\mathcal{O}[H])} \cong \operatorname{Pic}(\mathcal{O}[H])
$$

denote the natural quotient map (cf. Proposition 1.3). The following theorem gives the decomposition of a tame global resolvend. It is proved in exactly the same manner as Theorem 6.7 of [M] (and thus depends crucially upon Theorem 5.4).

Theorem 5.5. (Global Decomposition Theorem) Let $h \in \operatorname{Hom}\left(\Omega_{K}, H\right)$, and let $\alpha_{h}: Y_{h} \rightarrow X$ denote the corresponding Galois cover of $X$. Suppose that $K[H] . b=$ $K_{h}$. Then $h$ is tame if and only if there are elements $c \in J_{K}(K[H]), f \in F_{X, H}$ and $u \in \mathcal{H}(\mathbb{A}(\mathcal{O}[H]))$ such that

$$
\lambda\left(r_{H}(b)\right)=\left(\operatorname{rag}_{H}(c)\right)^{-1} \Theta_{H, \mu}^{t}(f) \cdot u
$$

Furthermore, if this is the case then $\rho(c)$ is the class of $\alpha_{h},{ }_{*} \mathcal{O}_{Y_{h}}$ in $\operatorname{Pic}(\mathcal{O}[H])$ and $f$ is unique. More precisely, we have $f=\left(f_{x}\right)_{x}$ where, for each $x \in X_{0}, f_{x}=f_{x, s}$ with $s=h_{x}\left(\sigma_{x}\right)$. In particular, therefore, $f_{x} \neq 1$ if and only if $h_{x}$ is ramified, and so $f=1$ if and only if $h$ is unramified. 
We can now give the following characterization of realizable classes in $\operatorname{Pic}(\mathcal{O}[H])$.

THEOREM 5.6. Suppose that $c \in J_{K}(K[H])$. Then

(i) $\quad \rho(c) \in \Re_{X, 0}^{0}(H)$ if and only if $\operatorname{rag}_{H}(c) \in \lambda(\mathcal{H}(K[H])) . \mathcal{H}(\mathbb{A}(\mathcal{O}[H]))$;

(ii) $\rho(c) \in \Re_{X}^{0}(H)$ if and only if $\operatorname{rag}_{H}(c) \in \lambda(\mathcal{H}(K[H]))$

. $\mathcal{H}(\mathbb{A}(\mathcal{O}[H])) \Theta_{H, \mu}^{t}\left(F_{X, H}\right)$.

Proof. Suppose now that $h \in \operatorname{Hom}\left(\Omega_{K}^{t}, H\right)$, and let $\alpha_{h}: Y_{h} \rightarrow X$ denote the corresponding Galois $H$-cover of $X$. Suppose further that $\rho(c)$ is equal to the class of $\alpha_{h},{ }_{*} \mathcal{O}_{Y_{h}}$ in $\operatorname{Pic}(\mathcal{O}[H])$. Let $K[H] . b=K_{h}$. Then it follows from Theorem 5.5 that there exist elements $c^{\prime} \in J_{K}(K[H]), f \in F_{X, H}$, and $u \in \mathcal{H}(\mathbb{A}(\mathcal{O}[H]))$ for which

$$
\rho(c)=\rho\left(c^{\prime}\right)
$$

and

$$
\lambda\left(r_{H}(b)\right)=\left(\operatorname{rag}_{H}\left(c^{\prime}\right)\right)^{-1} \Theta_{H, \mu}^{t}(f) . u,
$$

(with $f=1$ if and only if $h$ is unramified). Hence we have

$$
\operatorname{rag}_{H}\left(c^{\prime}\right) \in \lambda(\mathcal{H}(K[H])) . \mathcal{H}(\mathbb{A}(\mathcal{O}[H]))
$$

if $h$ is unramified, and more generally

$$
\operatorname{rag}_{H}\left(c^{\prime}\right) \in \lambda(\mathcal{H}(K[H])) \mathcal{H}(\mathbb{A}(\mathcal{O}[H])) \Theta_{H, \mu}^{t}\left(F_{X, H}\right)
$$

Now $c^{-1} c^{\prime} \in(K[H])^{*} U(\mathcal{O}[H])$ and so it follows that

$$
\operatorname{rag}_{H}\left(c^{-1} c^{\prime}\right) \in \lambda(\mathcal{H}(K[H])) \mathcal{H}(\mathbb{A}(\mathcal{O}[H]))
$$

This proves the "only if" parts of (i) and (ii).

Suppose conversely that $\operatorname{rag}_{H}(c) \in \lambda(\mathcal{H}(K[H])) \mathcal{H}(\mathbb{A}(\mathcal{O}[H])) \Theta_{H, \mu}^{t}\left(F_{X, H}\right)$. Then we have that

$$
\operatorname{rag}_{H}(c)=\lambda\left(r_{H}\left(b_{1}\right)\right)^{-1} u_{1} \Theta_{H, \mu}^{t}\left(f_{1}\right)
$$

where $K[H] . b_{1}=K_{h}$ for some $h \in \operatorname{Hom}\left(\Omega_{K}, H\right), u_{1} \in \mathcal{H}(\mathbb{A}(\mathcal{O}[H]))$ and $f_{1} \in$ $F_{X, H}$.

If $f=1$ then Theorem 5.5 implies that $h$ is unramified and that $\rho(c)$ is the class of $\mathcal{O}_{Y_{h}}$. This completes the proof of (i). The "if" part of (ii) is on the other hand an immediate consequence of Theorem 5.5. 
We note that, in the statement of Theorem 5.6 (ii), the subgroup $\Theta_{H, \mu}^{t}\left(F_{X, H}\right)$ is actually independent of the choice of $\mu$ (as a consequence of (5.8)).

We shall now derive the assertions of Theorem 2.9 (i) and (ii) as a consequence of Theorem 5.6. To do this we observe that the homomorphism

$$
\operatorname{rag}_{H}: J_{K}(K[H]) \rightarrow \mathcal{H}(\mathbb{A}(K[H]))
$$

induces upon restriction to augmentation a homomorphism

$$
\begin{aligned}
\operatorname{Rag}_{H}: \operatorname{Pic}(\mathcal{O}[H]) & \cong \operatorname{Hom}_{\Omega_{K}}\left(R_{H}, \operatorname{Pic}\left(K^{\prime}\right)\right) \cong \frac{J_{K}(K[H])}{(K[H])^{*} U(\mathcal{O}[H])} \\
& \left.\rightarrow \frac{\mathcal{H}(\mathbb{A}(K[H]))}{\lambda(\mathcal{H}(K[H])) \cdot \mathcal{H}(\mathbb{A}(\mathcal{O}[H]))} \cong \operatorname{Hom}_{\Omega_{K}}\left(A_{\hat{H}}, \operatorname{Pic} K^{\prime}\right)\right),
\end{aligned}
$$

whilst the map $\Theta_{H, \mu}^{t}: J_{K}(K \Lambda) \rightarrow \mathcal{H}(\mathbb{A}(K[H]))$ induces a map

$$
\Theta_{H, \mu}^{t}: \operatorname{Hom}_{\Omega_{K}}\left(\mathbb{Z}[H](\Leftrightarrow 1), \operatorname{Pic}\left(K^{\prime}\right)\right) \rightarrow \operatorname{Hom}_{\Omega_{K}}\left(A_{\hat{H}}, \operatorname{Pic}\left(K^{\prime}\right)\right) .
$$

The result of Theorem 2.9 (i) and (ii) is thus simply the translation of Theorem 5.6 via these homomorphisms into the Hom-description of Proposition 1.3.

Remark 5.7. Note that if $f \in F_{X, H}$ and $c \in \operatorname{Rag}_{H}^{-1}\left(\Theta_{H, \mu}^{1}(f)\right)$ then $\operatorname{deg}(c(\chi)) \geq$ 0 for all characters $\chi \in \hat{H}$. Furthermore, $\operatorname{deg}(c(\chi))=0$ for all characters $\chi$ if and only if $f=1$, that is, if and only if the corresponding Galois cover of $X$ is étale. Hence, in general, $\Re_{X}^{0}(H)$ is not a subgroup of Pic $(\mathcal{O}[H])$ (cf. Remark 2.10 (iii)).

6. Classes arising from general invertible sheaves. In this section we shall prove parts (iii) and (iv) of Theorem 2.9, the notation of which we shall continue to use. The argument given here is modelled on that of [Bu].

We shall first show that

$$
\Re_{X}(H) \subseteq\left\langle\Re_{X}^{0}(H)\right\rangle+\sum_{C \in \mathcal{C}(H)} \operatorname{ind}_{C}^{H} \operatorname{Pic}^{\# C}(\mathcal{O}[C])
$$

and that

$$
\Re_{X, 0}(H) \subseteq \Re_{X, 0}^{0}(H)+\operatorname{ind}_{\{1\}}^{H} \operatorname{Pic}(\mathcal{O})
$$

These inclusions will be seen to be a consequence of the functorial behavior of Chapman's Hom-description (cf. Proposition 1.3 and Lemma 1.4) together with some standard properties of tame local extensions.

We suppose then that the class $\mathfrak{c} \in \operatorname{Pic}(\mathcal{O}[H])$ is realizable, corresponding to a Galois $H$-cover $f: Y \rightarrow X$ of $X$, an identification of $\operatorname{Gal}(Y / X)$ with $H$ (which 
we henceforth suppress) and a $H$-stable invertible $\mathcal{O}_{Y}$-sheaf $\mathcal{A}$. Since

$$
\mathfrak{c}=\left(f_{*} \mathcal{A}\right)=\left(f_{*} \mathcal{O}_{Y}\right)+\left(\left(f_{*} \mathcal{A}\right) \Leftrightarrow\left(f_{*} \mathcal{O}_{Y}\right)\right) \in \operatorname{Pic}(\mathcal{O}[H])
$$

and obviously $\left(f_{*} \mathcal{O}_{Y}\right) \in \Re_{X}^{0}(H)$, respectively $\left(f_{*} \mathcal{O}_{Y}\right) \in \Re_{X, 0}^{0}(H)$ if $f$ is étale, we shall only need to show that the class $\mathfrak{c}^{\prime}:=\left(f_{*} \mathcal{A}\right) \Leftrightarrow\left(f_{*} \mathcal{O}_{Y}\right)$ satisfies

$$
\mathfrak{c}^{\prime} \in \sum_{C \in \mathcal{C}(H)} \operatorname{ind}_{C}^{H} \operatorname{Pic}^{\# C}(\mathcal{O}[C])
$$

respectively

$$
\mathfrak{c}^{\prime} \in \operatorname{ind}_{\{1\}}^{H} \operatorname{Pic}(\mathcal{O})
$$

if $f$ is étale. We shall prove these using the Galois resolvent techniques discussed by Chapman in [C].

For each field $F \subset K^{c}$ and each character $\chi \in \hat{H}$ we let $F_{\chi}$ denote the field extension of $F$ generated by adjoining the values of $\chi$. For each point $x \in X$ we choose a free $\mathcal{O}_{X}[H]$-generator $a_{1, x}$, respectively $a_{2, x}$, for the stalk $\mathcal{A}_{x}$, respectively $\mathcal{O}_{Y, x}$. For each $x \in X$, each $\chi \in \hat{H}$, and both $i=1$ and $i=2$, we define a Galois resolvent

$$
\left(a_{i, x} \mid \chi\right):=\sum_{h \in H} h\left(a_{i, x}\right) \chi\left(h^{-1}\right) \in K^{c *}
$$

The quotient $\left(a_{1, x} \mid \chi\right) /\left(a_{2, x} \mid \chi\right)$ belongs to $K_{\chi}$. Furthermore, as a simple consequence of the considerations of ([C], pp. 21-23) one knows that under the isomorphism $\theta_{\mathcal{O}[H]}$ of Proposition 1.3 the class $\mathfrak{c}^{\prime}$ corresponds to the function $g \in \operatorname{Hom}_{\Omega_{K}}\left(R_{H}, \operatorname{Div}\left(K^{\prime}\right)\right)$ which is defined at each character $\chi \in \hat{H}$ by

$$
g(\chi):=\sum_{\mathrm{v}^{\prime}} \operatorname{Val}_{\mathrm{V}^{\prime}}\left(\frac{\left(a_{1, \mathrm{v}\left(\mathrm{v}^{\prime}\right)} \mid \chi\right)}{\left(a_{2, \mathrm{v}\left(\mathrm{v}^{\prime}\right)} \mid \chi\right)}\right) \mathrm{v}^{\prime} \in \operatorname{Div}\left(K_{\chi}\right)
$$

where here $\mathrm{v}^{\prime}$ runs over the prime divisors of $K_{\chi}, \mathrm{Val}_{\mathrm{V}^{\prime}}$ denotes the valuation of $K_{\chi}$ corresponding to $\mathrm{v}^{\prime}$, and $\mathrm{v}\left(\mathrm{v}^{\prime}\right)$ is the prime divisor of $K$ lying beneath $\mathrm{v}^{\prime}$. We write $\operatorname{Supp}\left(\mathcal{O}_{Y}, \mathcal{A}\right)$ for the set of prime divisors of $K$ at which the stalks of $f_{*} \mathcal{O}_{Y}$ and $f_{*} \mathcal{A}$ differ. For each $\mathrm{v} \in \operatorname{Supp}\left(\mathcal{O}_{Y}, \mathcal{A}\right)$ we let $g_{\mathrm{v}} \in \operatorname{Hom}_{\Omega_{K}}\left(R_{H}, \operatorname{Div}\left(K^{\prime}\right)\right)$ be defined at each character $\chi \in \hat{H}$ by

$$
g_{\mathrm{v}}(\chi):=\sum_{\mathrm{v}^{\prime} \mid \mathrm{v}} \operatorname{Val}_{\mathrm{V}^{\prime}}\left(\frac{\left(a_{1, \mathrm{v}} \mid \chi\right)}{\left(a_{2, \mathrm{v}} \mid \chi\right)}\right) \mathrm{v}^{\prime} \in \operatorname{Div}\left(K_{\chi}\right)
$$

where here the summation is taken over all prime divisors $\mathrm{v}^{\prime}$ of $K_{\chi}$ which lie 
above v. It is clear that

$$
g=\sum_{\mathrm{v} \in \operatorname{Supp}\left(\mathcal{O}_{Y}, \mathcal{A}\right)} g_{\mathrm{v}}
$$

For each $\mathrm{v} \in \operatorname{Supp}\left(\mathcal{O}_{Y}, \mathcal{A}\right)$ we let $I(\mathrm{v})$ denote the inertial subgroup of $\mathrm{v}$ in $H$.

LEMMA 6.1. For each prime divisor $\mathrm{v} \in \operatorname{Supp}\left(\mathcal{O}_{Y}, \mathcal{A}\right)$ one has $g_{\mathrm{v}}=\tilde{g}_{\mathrm{v}} \circ \operatorname{res}_{I(\mathrm{v})}^{H}$ for a function $\tilde{g}_{\mathrm{v}} \in \operatorname{Hom}_{\Omega_{K}}\left(R_{I(\mathrm{v})}\right.$, Div $\left.\left(K^{\prime}\right)\right)$ which is supported entirely above $\mathrm{v}$.

Before proving this lemma we note that it is sufficient to prove the desired inclusions (6.3) and (6.4). To see this we note firstly that since $\mathrm{V}$ is tamely ramified in $K_{Y} / K$ the inertial subgroup $I(\mathrm{v})$ is cyclic. In addition, since $H$ is abelian the tameness of $\mathrm{v}$ in $K_{Y} / K$ also implies that the norm of $\mathrm{v}$ is congruent to 1 modulo \#I(v) and so Lemma 6.1 implies that $\tilde{g}_{\mathrm{v}}$ represents a class in $\operatorname{Pic}^{\# I(\mathrm{v})}(\mathcal{O}[I(\mathrm{v})])$. Finally we use the equality $g_{\mathrm{v}}=\tilde{g}_{\mathrm{V}} \circ \operatorname{res}_{I(\mathrm{v})}^{H}$ of Lemma 6.1 in conjunction with Lemma 1.4 to deduce that $g_{\mathrm{v}}$ represents a class in $\operatorname{ind}_{I(\mathrm{v})}^{H} \operatorname{Pic}^{\# I(\mathrm{v})}(\mathcal{O}[I(\mathrm{v})])$. The inclusions (6.3) and (6.4) are therefore an immediate consequence of the decomposition (6.6).

Proof of Lemma 6.1. It is sufficient for us to show that for each prime divisor $\mathrm{v}^{\prime}$ of $K_{\chi}$ over $\mathrm{v}$ the valuation $\operatorname{Val}_{\mathrm{v}^{\prime}}\left(\left(a_{1, \mathrm{v}} \mid \chi\right) /\left(a_{2, \mathrm{v}} \mid \chi\right)\right)$ depends, as a function of $\chi$, only upon the restricted character $\operatorname{res}_{I(\mathrm{v})}^{H} \chi$. Indeed, if this is the case then we can define a function $\tilde{g}_{\mathrm{v}} \in \operatorname{Hom}_{\Omega_{K}}\left(R_{I(\mathrm{v})}\right.$, Div $\left.\left(K^{\prime}\right)\right)$ by setting for each $\phi \in I(\mathrm{v})^{\wedge}$

$$
\tilde{g}_{\mathrm{v}}(\phi):=g_{\mathrm{v}}(\chi) \quad \text { if } \quad \chi \in \hat{H} \quad \text { and } \quad \operatorname{res}_{I(\mathrm{v})}^{H} \chi=\phi,
$$

and then obviously $g_{\mathrm{v}}=\tilde{g}_{\mathrm{v}} \circ \operatorname{res}_{I(\mathrm{v})}^{H}$.

To proceed, set $N=K_{Y}$ and let $E$ denote the compositum $N K_{\chi}$. Fix an embedding $j: E \hookrightarrow K_{\mathrm{V}}^{c}$ such that $\left.j\right|_{K_{\chi}}$ corresponds to $\mathrm{V}^{\prime}$, and let $w$ be the prime divisor of $N$ which corresponds to the restriction $\left.j\right|_{N}$. We let $N_{w, 0}$ denote the maximal unramified extension of $K_{\mathrm{V}}$ in $N_{w}$, and we identify the groups $\operatorname{Gal}\left(N_{w} / N_{w, 0}\right)$ and $I(\mathrm{v})$ via the embedding $\left.j\right|_{N}$. With $\mathcal{O}_{0}$ denoting the valuation ring of $N_{w, 0}$ one knows that the stalks $\mathcal{A}_{w}$ and $\mathcal{O}_{Y, w}$ are free $\mathcal{O}_{0}[I(\mathrm{v})]$-modules. Furthermore, from the arguments of Theorem 19 and Theorem 25 (ii) of [F] (cf. also [Bu], (2.6-7)) one knows that for any choice of free $\mathcal{O}_{0}[I(\mathrm{v})]$-generators $c_{1, w}$, respectively $c_{2, w}$, of $\mathcal{A}_{w}$, respectively $\mathcal{O}_{Y, w}$, there is a unit $\lambda$ of the valuation ring of the closure of $j E$ in $K_{\mathrm{v}}^{c}$ such that

$$
j\left(\frac{\left(a_{1, \mathrm{v}} \mid \chi\right)}{\left(a_{2, \mathrm{v}} \mid \chi\right)}\right)=\frac{\left(c_{1, w} \mid \operatorname{res}_{I(\mathrm{v})}^{H}(j \circ \chi)\right)}{\left(c_{2, w} \mid \operatorname{res}_{I(\mathrm{v})}^{H}(j \circ \chi)\right)} \lambda .
$$

By taking $\mathrm{V}^{\prime}$-valuations of this equation we see that the $\mathrm{V}^{\prime}$-valuation of $\left(a_{1, \mathrm{v}}\right)$ $\chi) /\left(a_{2, \mathrm{v}} \mid \chi\right)$ depends as a function of $\chi$ only upon the restricted character $\operatorname{res}_{I(\mathrm{v})}^{H} \chi$. 
Having proved (6.1) and (6.2) our proof of parts (iii) and (iv) of Theorem 2.9 will be complete if we can prove that

$$
\left\langle\Re_{X}^{0}(H)\right\rangle+\sum_{C \in \mathcal{C}(H)} \operatorname{ind}_{C}^{H} \operatorname{Pic}^{\# C}(\mathcal{O}[C]) \subseteq \Re_{X}(H),
$$

and

$$
\Re_{X, 0}^{0}(H)+\operatorname{ind}_{\{1\}}^{H} \operatorname{Pic}(\mathcal{O}) \subseteq \Re_{X, 0}(H)
$$

We shall deal firstly with the inclusion (6.8). To prove this it is obviously sufficient to show that $\operatorname{ind}_{C}^{H} \operatorname{Pic}^{\# C}(\mathcal{O}[C]) \subseteq \Re_{X}(H)$ for any cyclic subgroup $C$ of $H$. To this end we henceforth fix a cyclic subgroup $C$ of $H$ and set $n=\# C$. For each character $\chi \in \hat{C}$ we let $C_{\chi}$ denote its kernel and set $n_{\chi}=\# C_{\chi}$. The field $K_{\chi}$ is thus a (constant) field extension of $K$ of degree $n / n_{\chi}$. We let $\mathcal{D}(C)$ denote the set of equivalence classes of $\hat{C}$ under the relation of $\Omega_{K}$-conjugacy. Since $K_{\chi}$ depends only upon the element $D=D_{\chi}$ of $\mathcal{D}(C)$ to which $\chi$ belongs we shall also denote it by $K_{D}$. One has

$$
\operatorname{Hom}_{\Omega_{K}}\left(R_{C}, \operatorname{Pic}^{n}\left(K^{\prime}\right)\right) \cong \bigoplus_{D \in \mathcal{D}(C)} \operatorname{Hom}_{\Omega_{K}}\left(\mathbb{Z}[D], \operatorname{Pic}^{n}\left(K_{D}\right)\right),
$$

and so we shall need to show that

$$
\sum_{D \in \mathcal{D}(C)} \operatorname{ind}_{C}^{H} \operatorname{Hom}_{\Omega_{K}}\left(\mathbb{Z}[D], \operatorname{Pic}^{n}\left(K_{D}\right)\right) \subseteq \theta_{\mathcal{O}[H]} \Re_{X}(H) .
$$

The first step in verifying this inclusion is for us to observe that each group $\mathrm{Pic}^{n}\left(K_{D}\right)$ is generated by the classes of prime divisors.

LEMMA 6.2. For each class $D \in \mathcal{D}(C)$, and for each strictly positive integer $N$, the group $\mathrm{Pic}^{N}\left(K_{D}\right)$ is generated by the classes of prime divisors.

Proof. For this argument we let $P \operatorname{Pic}^{N}\left(K_{D}\right)$ denote the subgroup of $\operatorname{Pic}^{N}\left(K_{D}\right)$ which is generated by the classes of those prime divisors which belong to $\operatorname{Div}^{N}\left(K_{D}\right)$. If $p \mid N$ then $\operatorname{Pic}^{N}\left(K_{D}\right)=0$ and so the lemma is obviously true in this case. We therefore suppose that $p \nmid N$. In this case, one knows (for example, as a consequence of the Weil estimate for the number of points on a variety over a finite field) that there exist prime divisors on $K_{D}$ which have norm congruent to 1 modulo $N$, and hence the subgroups $\operatorname{Pic}^{N}\left(K_{D}\right)$ and $P \operatorname{Pic}^{N}\left(K_{D}\right)$ are both of finite index in Pic $\left(K_{D}\right)$. If $H$ is the Hilbert class field of $K_{D}$ the from global class field theory it follows that there are fields $L_{1}$ and $L_{2}$, corresponding to the groups $\operatorname{Pic}^{N}\left(K_{D}\right)$ and $P \operatorname{Pic}^{N}\left(K_{D}\right)$ respectively, which are such that $K_{D} \subseteq L_{1} \subseteq L_{2} \subseteq H$. The prime divisors of $K_{D}$ which split completely in $L_{1} / K_{D}$ are precisely those which have class in $\mathrm{Pic}^{N}\left(K_{D}\right)$, and so coincide with those prime divisors of $K_{D}$ 
which split completely in $L_{2} / K_{D}$. Using this fact the Tchebatarev theorem implies that $L_{1}=L_{2}$, and from this it follows that $P \operatorname{Pic}^{N}\left(K_{D}\right)=\operatorname{Pic}^{N}\left(K_{D}\right)$.

The set of subgroups of $C$ corresponds bijectively to the set of positive divisors of $n$ (as $C$ is cyclic) and so is linearly ordered. Since the group $\Re_{X}(H)$ consists of arbitrary finite linear combinations of realizable elements, this means that (6.10) can be obtained as a consequence of Lemma 6.2 taken in conjunction with the following result.

Proposition 6.3. Let $\chi_{0} \in \hat{C}$, with $\chi_{0} \in D_{0}$ say, and choose a prime divisor $\wp$ of $K_{D_{0}}$ which has norm congruent to 1 modulo $n$. Then $\theta_{\mathcal{O}[H]} \Re_{X}(H)$ contains an element of the form ind ${ }_{C}^{H} g$ for some function $g \in \operatorname{Hom}_{\Omega_{K}}\left(R_{C}\right.$, $\left.\operatorname{Pic}^{n}\left(K^{\prime}\right)\right)$ which satisfies the following property: for each character $\chi \in \hat{C}$ for which $n_{\chi} \geq n_{\chi_{0}}$ one has

$$
g(\chi)= \begin{cases}\wp, & \text { if } \chi=\chi_{0} \\ 0, & \text { if } D_{\chi} \neq D_{0}\end{cases}
$$

To prove this proposition we shall simply construct Galois $H$-covers of $X$ which possess $H$-stable invertible sheaves which give rise to classes represented by the kind of function described in the above statement.

We now fix a character $\chi_{0}$ and a prime divisor $\wp$ as in the statement of Proposition 6.3, and we let $\mathfrak{p}$ denote the prime divisor of $K$ lying beneath $\wp$. We choose a maximal cyclic subgroup $C^{\prime}$ of $H$ which contains $C$, and a subgroup $J$ of $H$ for which $H=C^{\prime} \times J$ (such a subgroup $J$ must exist). Since $\mathfrak{p}$ has norm congruent to 1 modulo $n$ the Grunwald-Wang theorem (cf. [Ar,T], Chapter 10, $\S 2$ ) allows us to choose a cyclic extension $F$ of $K$ of degree $\# C^{\prime}$ in which the inertial and decomposition subgroups of $\mathfrak{p}$ coincide, and are of order $n$. By again using the Grunwald-Wang theorem we may choose a Galois extension $F_{1}$ of $K$ which is disjoint from $F / K$, has group isomorphic to $J$, and in which $\mathfrak{p}$ splits completely. We fix an identification of $\operatorname{Gal}\left(F_{1} / K\right)$ with $J$. We let $N$ denote the compositum $F F_{1}$, and next specify an identification of $\mathrm{Gal}(F / K)$ with $C^{\prime}$, and hence of

$$
\operatorname{Gal}(N / K) \cong \operatorname{Gal}\left(N / F_{1}\right) \times \operatorname{Gal}(N / F) \cong \operatorname{Gal}(F / K) \times \operatorname{Gal}\left(F_{1} / K\right)
$$

with $H$. For this we let $M$ denote the subfield of $F$ which corresponds to the kernel of any element of $D_{0}$, with $L$ the maximal extension of $K$ in $F$ in which $\mathfrak{p}$ is unramified. Thus $L \subseteq M$ and $\operatorname{Gal}(F / L) \cong C$. We write $K_{0}$ for the completion of $K$ at $\mathfrak{p}$, choose and fix an embedding $j_{0}: K^{c} \hookrightarrow K_{0}^{c}$ which corresponds on $K_{D_{0}}$ to $\wp$, and we write $M_{0}$ for the completion of $M$ at the place corresponding to $j_{0}$. The completion of $L$ at the place corresponding to $j_{0}$ is $K_{0}$ and so we can identify $\operatorname{Gal}(M / L)$ and $\operatorname{Gal}\left(M_{0} / K_{0}\right)$ via the embedding $\left.j_{0}\right|_{M}$.

To proceed further we must now recall some basic facts concerning 
the arithmetic of $M_{0} / K_{0}$. We recall first that since $M_{0} / K_{0}$ is totally and tamely ramified each element of $\operatorname{Gal}\left(M_{0} / K_{0}\right)^{\wedge}$ is valued in $K_{0}^{*}$. In addition, if for each character $\chi \in \operatorname{Gal}\left(M_{0} / K_{0}\right)^{\wedge}$ we write $e_{\chi}$ for the idempotent $\# \operatorname{Gal}\left(M_{0} / K_{0}\right)^{-1} \sum_{g \in \operatorname{Gal}\left(M_{0} / K_{0}\right)} \chi\left(g^{-1}\right) g$ of $\mathcal{O}_{K}\left[\mathrm{Gal}\left(M_{0} / K_{0}\right)\right]$ and we let $\operatorname{Val}_{0}$ denote the standard valuation on $M_{0}$, then the group $\operatorname{Gal}\left(M_{0} / K_{0}\right)^{\wedge}$ possesses a cononical generator $\chi_{M_{0} / K_{0}}$ which is such that for each $x \in M_{0}$ and $\chi \in$ $\operatorname{Gal}\left(M_{0} / K_{0}\right)^{\wedge}$ for which $e_{\chi} x \neq 0$ has

(6.11) $\operatorname{Val}_{0}\left(e_{\chi} x\right) \geq \operatorname{Val}_{0}(x), \quad$ with equality here if and only if $\chi=\chi_{M_{0} / K_{0}}^{\operatorname{Val}_{0}(x)}$

(cf. Proposition 1 of [Be]). Now any isomorphism between $\mathrm{Gal}(F / K)$ and $C^{\prime}$ induces an isomorphism between $\operatorname{Gal}(F / L)$ and $C$, and so allows us to regard $\chi_{0}$ as an element of $\operatorname{Gal}(M / L)^{\wedge}=\operatorname{Gal}\left(M_{0} / K_{0}\right)^{\wedge}$. We choose an isomorphism between $\mathrm{Gal}(F / K)$ and $C^{\prime}$ which is such that, upon regarding $\chi_{0}$ as a character of $\operatorname{Gal}\left(M_{0} / K_{0}\right)$ via this isomorphism, one has $j_{0} \circ \chi_{0}=\chi_{M_{0} / K_{0}}$ (it is not difficult to see that such a choice of isomorphism must exist).

For each intermediate field $N^{\prime}$ of $N / K$ we write $f_{N^{\prime}}: Y\left(N^{\prime}\right) \rightarrow X$ for the corresponding cover of curves, and we let $\mathcal{P}\left(N^{\prime}\right)$ denote the invertible $\mathcal{O}_{Y\left(N^{\prime}\right)^{-}}$ sheaf which corresponds to the divisor of $N^{\prime}$ obtained by summing over all prime divisors lying over $\mathfrak{p}$.

We set $f=f_{N}$, and for each integer $m$, and each point $x$ of $X$, we choose a free $\mathcal{O}_{x}[H]$-generator $a_{m, x}$ of stalk $f_{*}\left(\mathcal{P}(N)^{m}\right)_{x}$. For each pair of integers $m$ and $n$ we define a function $g_{m, n}$ on $\hat{H}$ by setting

$$
g_{m, n}(\chi):=\sum_{\mathrm{V}^{\prime} \mid \mathfrak{p}} \operatorname{Val}_{\mathrm{V}^{\prime}}\left(\frac{\left(a_{n, \mathfrak{p}} \mid \chi\right)}{\left(a_{m, \mathfrak{p}} \mid \chi\right)}\right) \mathrm{v}^{\prime} \in \operatorname{Div}\left(K_{\chi}\right)
$$

where here the sum is over all prime divisors $\mathrm{v}^{\prime}$ of $K_{\chi}$ lying over $\mathfrak{p}$. Note in particular that under the Hom-description used above (cf. (6.5)) the function $g_{m, n}$ represents the image under $\theta_{\mathcal{O}[H]}$ of the class

$$
c(m, n):=\left(f_{*} \mathcal{P}(N)^{n}\right) \Leftrightarrow\left(f_{*} \mathcal{P}(N)^{m}\right) \in \operatorname{Pic}(\mathcal{O}[H])
$$

We let $f^{\prime}: Y(F) \rightarrow Y(L)$ be the morphism of curves induced by $f_{F}$. For each point $y$ of $Y(L)$, and each integer $m$, we choose a free $\mathcal{O}_{Y(L), y}[C]$-generator $b_{m, y}$ of the stalk $f_{*}^{\prime}\left(\mathcal{P}(F)^{m}\right)_{y}$. We define a function $g_{m, n}^{*}$ on $\hat{C}$ by setting

$$
g_{m, n}^{*}(\chi):=\sum_{s^{\prime} \mid \mathfrak{p}} \operatorname{Val}_{s^{\prime}}\left(\frac{\left(b_{m, s\left(s^{\prime}\right)} \mid \chi\right)}{\left(b_{n, s\left(s^{\prime}\right)} \mid \chi\right)}\right) s^{\prime} \in \operatorname{Div}\left(L_{\chi}\right)
$$

where here $s^{\prime}$ runs over the prime divisors of $L_{\chi}$ lying above $\mathfrak{p}$ and $s\left(s^{\prime}\right)$ is the 
prime divisor of $L$ which lies below $s^{\prime}$. The fact that $\mathfrak{p}$ is chosen to split in the extension $F_{1} L / K$ is now reflected in the following result.

LEMMA 6.4. For each character $\chi \in \hat{H}$ one has

$$
g_{m, n}(\chi)=g_{m, n}^{*}\left(\operatorname{res}_{C}^{H} \chi\right) \in \operatorname{Div}\left(L_{\chi}\right) .
$$

Proof. We choose an embedding $j: N K_{\chi} \hookrightarrow K_{\mathfrak{p}}^{c}$, and let $w, \mathrm{v}^{\prime}, t, s^{\prime}$ and $s$ denote the prime divisors of $N, K_{\chi}, F, L_{\chi}$, and $L$ which correspond to the respective restrictions of $j$.

The maximal unramified extension of $K_{\mathfrak{p}}$ in $N_{w}$ is $K_{\mathfrak{p}}$ itself, and so the argument leading to (6.7) here implies that the coefficient of $\mathrm{v}^{\prime}$ in the divisor $g_{m, n}(\chi) \in \operatorname{Div}\left(K_{\chi}\right)$ is equal to the $\mathrm{v}^{\prime}$-valuation

$$
\operatorname{Val}_{\mathrm{V}^{\prime}}\left(\frac{\left.\left(c_{n, w} \mid \operatorname{res}_{I \mathfrak{p}}^{H}\right)(j \circ \chi)\right)}{\left.\left(c_{m, w} \mid \operatorname{res}_{I \mathfrak{p}}^{H}\right)(j \circ \chi)\right)}\right)
$$

where here $c_{m, w}$ and $c_{n, w}$ are free $\mathcal{O}_{\mathfrak{p}}[I(\mathfrak{p})]$-generators of the stalks $\left(\mathcal{P}(N)^{m}\right)_{w}$ and $\left(\mathcal{P}(N)^{n}\right)_{w}$ respectively.

Under our chosen identification of $H$ with $\operatorname{Gal}(N / K)$ we have $C=I(\mathfrak{p}) \leq$ $\mathrm{Gal}(N / K)$. In addition, since $\mathfrak{p}$ splits completely in $F_{1} L / K$ there are identifications $N_{w}=F_{t},\left(\mathcal{P}(N)^{m}\right)_{w}=\left(\mathcal{P}(F)^{m}\right)_{t}$ for each integer $m$, and $L_{s}=K_{\mathfrak{p}}$. Just as above, we can deduce that the coefficient of $s^{\prime}$ in the divisor $g_{m, n}^{*}\left(\operatorname{res}_{C}^{H} \chi\right) \in$ $\operatorname{Div}\left(L_{\chi}\right)$ is equal to the $s^{\prime}$-valuation

$$
\operatorname{Val}_{s^{\prime}}\left(\frac{\left(c_{n, w} \mid \operatorname{res}_{I(\mathfrak{p})}^{H}(j \circ \chi)\right)}{\left(c_{m, w} \mid \operatorname{res}_{I(\mathfrak{p})}^{H}(j \circ \chi)\right)}\right) .
$$

Since $L_{\chi, s^{\prime}}=K_{\chi, \mathrm{v}^{\prime}}$ this last expression is equal to (6.12), and the claimed equality of divisors now follows immediately from this.

From Lemma 1.4 (with $G=H$ and $J=C$ ) and Lemma 6.4 it follows that

$$
c(m, n)=\operatorname{ind}_{C}^{H} \hat{c}(m, n)
$$

with $\hat{c}(m, n) \in \operatorname{Pic}(\mathcal{O}[C])$ the class whose image under $\theta_{\mathcal{O}[C]}$ corresponds to the function $g_{m, n}^{*}$. We shall thus obtain a proof of Proposition 6.3 by choosing integers $m$ and $n$ according to the following lemma.

LEMMA 6.5. For each character $\chi \in \hat{C}$ for which $n_{\chi} \geq n_{\chi_{0}}$ one has

$$
g_{1, n_{\chi_{0}}+1}^{*}(\chi)= \begin{cases}\wp, & \text { if } \chi=\chi_{0} \\ 0, & \text { if } \chi \in D_{0}\end{cases}
$$


Proof. We shall use the same notation as in the proof of Lemma 6.4. In addition, for each $\chi \in C$ we let $C_{\chi}$ denote the kernel of $\chi$, and we set $F^{\chi}=F^{C_{\chi}}$, $n_{\chi}=\# C_{\chi}$ with $n_{0}=n_{\chi_{0}}$, and $\sigma_{\chi}=\sum_{c \in C_{\chi}} c \in \mathbb{Z}\left[C_{\chi}\right]$. We let $\operatorname{Val}_{\chi}(\Leftrightarrow)$ denote the standard valuation on $F^{\chi}$, and we write $\bar{\chi}$ for the element of $\left(C / C_{\chi}\right)^{\wedge}$ induced by $\chi$.

For each integer $m$ one has

$$
\left(c_{m, w} \mid j \circ \chi\right)=\left(n_{\chi}\right)^{-1} e_{j \circ \bar{\chi}}\left(\sigma_{\chi} c_{m, w}\right),
$$

and as a consequence of (6.11) one knows that the valuation of this element depends only upon the character $j \circ \bar{\chi}$ and the valuation $\operatorname{Val}_{\chi}\left(\sigma_{\chi} c_{m, w}\right)$. Now if $n_{\chi}>n_{0}$ then $\operatorname{Val}_{\chi}\left(\sigma_{\chi} c_{1, w}\right)=\operatorname{Val}_{\chi}\left(\sigma_{\chi} c_{n_{0}+1, w}\right)=1$ and this quickly implies that $g_{1, n_{0}+1}^{*}(\chi)=0$. One needs slightly more of an argument to deal with characters $\chi$ for which $n_{\chi}=n_{0}$. Indeed, in this case $\operatorname{Val}_{\chi}\left(\sigma_{\chi} c_{1, w}\right)=1$ whilst $\operatorname{Val}_{\chi}\left(\sigma_{\chi} c_{n_{0}+1, w}\right)=$ 2. By using (6.11) one computes in this case that

$$
\operatorname{Val}_{\chi}\left(e_{j \circ \bar{\chi}}\left(\sigma_{\chi} c_{n_{0}+1, w}\right)\right)= \begin{cases}\operatorname{Val}_{\chi}\left(e_{j \circ \bar{\chi}}\left(\sigma_{\chi} c_{1, w}\right)\right), & \text { if } j \circ \bar{\chi} \neq \chi_{M_{0} / K_{0}} \\ \operatorname{Val}_{\chi}\left(e_{j \circ \bar{\chi}}\left(\sigma_{\chi} c_{1, w}\right)\right)+n / n_{\chi}, & \text { if } j \circ \bar{\chi}=\chi_{M_{0} / K_{0}},\end{cases}
$$

and this in turn implies that

$$
\operatorname{Val}_{s^{\prime}}\left(\frac{\left(c_{n_{0}+1, w} \mid j \circ \chi\right)}{\left(c_{1, w} \mid j \circ \chi\right)}\right)= \begin{cases}0, & \text { if } j \circ \bar{\chi} \neq \chi_{M_{0} / K_{0}} \\ 1, & \text { if } j \circ \bar{\chi}=\chi_{M_{0} / K_{0}} .\end{cases}
$$

This equality is enough to imply the stated result since, given our chosen identification of $C$ with $\operatorname{Gal}(F / L)$, one has $j_{0} \circ \overline{\chi_{0}}=\chi_{M_{0} / K_{0}}$ for an embedding $j_{0}: N K_{\chi_{0}} \hookrightarrow K_{\mathfrak{p}}^{c}$ which corresponds on $K_{\chi_{0}}$ to the prime divisor $\wp$.

We must finally check that (6.9) is true. To do this we choose for each class $\mathfrak{c} \in \operatorname{Pic}(\mathcal{O})$ a divisor $d \in \operatorname{Div}(K)$ which has class $\mathfrak{c}$. We let $f: Y \rightarrow X$ denote the trivial $H$-torsor over $X$, and write $\mathcal{D}_{Y}$ for the $\left(H\right.$-stable) invertible $\mathcal{O}_{Y}$-sheaf which corresponds to the divisor $d$. It is straightforward to check that the class of $\left(f_{*} \mathcal{D}_{Y}\right) \Leftrightarrow\left(f_{*} \mathcal{O}_{Y}\right)$ in $\operatorname{Pic}(\mathcal{O}[H])$ is equal to $\operatorname{ind}_{\{1\}}^{H} \mathfrak{c}$, and since $f$ is étale this proves (6.9).

DEPARTMENT OF MATHEMATICS, University OF CALIFORNiA, SANTA BARBARA, CA 93106

Electronic mail: AGBOOLA@MATH.UCSB.EDU

Department of Mathematics, King's College London, STRAnd, London WC2R 2LS, ENGLAND, U.K.

Electronic mail: DAVID.BURNS@KCL.AC.UK 


\section{REFERENCES}

[A,Bu] A. Agboola and D. Burns, Grothendieck groups of bundles on varieties over finite fields, preprint, 1997.

[Ar,T] E. Artin and J. Tate, Class Field Theory, Benjamin, New York, 1967.

[Ba] H. Bass, Algebraic K-theory, Benjamin, New York, 1968.

[Be] A.-M. Bergé, Arithmétique d'une extension galoisienne à groupe d'inertie cyclique, Ann. Inst. Fourier 28 (1978), 17-44.

[Bu] D. Burns, On arithmetically realisable classes, Math. Proc. Cambridge Philos. Soc. 118 (1995), 383-392.

[Bu,Ch] D. Burns and T. Chinburg, Adams operations and integral hermitian Galois representations, Amer. J. Math. 188 (1996), 925-962.

[By] N. P. Byott, Hopf orders and a generalisation of a theorem of L. R. McCulloh, J. Algebra 177 (1995), 409-433.

[C] R. Chapman, Class groups of sheaves of locally-free modules over global function fields, The Arithmetic of Function Fields (D. Goss, ed.), Ohio State Univ. Math. Res. Inst. Publ., vol. 2, de Gruyter, Berlin, 1992.

[Ch1] T. Chinburg, Galois module structure of de Rham cohomology, Sém. Théorie Nombres Bordeaux 4 (1991), 1-18.

[Ch2] Galois structure of de Rham cohomology of tame covers of schemes, Ann. of Math. 139 (1994), 443-490.

[Ch,E] T. Chinburg and B. Erez, Equivariant Euler-Poincaré Characteristics and Tameness, Proceedings of Journée Arithmétique 1991, Astérisque, Soc. Math. France, Paris, 1992.

[Ch,E,P,T] T. Chinburg, B. Erez, G. Pappas, and M. J. Taylor, Arithmetic equivariant Riemann-Roch theorems, preprint, 1993.

[EGA1] A. Grothendieck and J. Dieudonné, Eléments de Géométrie Algébrique I, Springer-Verlag, New York, 1971.

[E,L] G. Ellingsrud and K. Lonsted, An equivariant Lefschetz Formula for finite reductive groups, Math. Ann. 251 (1980), 253-261.

[F] A. Fröhlich, Galois Module Structure of Algebraic Integers, Ergeb. Math. Grenzgeb., SpringerVerlag, New York, 1983.

[F,L] W. Fulton and S. Lang, Riemann-Roch Algebra, Springer-Verlag, New York, 1985.

[SGA1] A. Grothendieck, Revetements Étales et Groupe Fondamental, Lecture Notes in Math., vol. 224, Springer-Verlag, New York, 1971.

[H] R. Hartshorne, Algebraic Geometry, Graduate Texts in Math., vol. 52, Springer-Verlag, New York, 1977.

[K] B. Köck, Adams operations for projective modules over group rings, Math. Proc. Cambridge Philos. Soc. 122 (1997), 55-71.

[L1] S. Lang, Algebra, 2nd ed., Addison-Wesley, Reading, MA, 1984.

[L2] Algebraic Number Theory, 2nd ed., Graduate Texts in Math., vol. 110, SpringerVerlag, New York, 1986.

[M] L. McCulloh, Galois module structure of abelian extensions, J. Reine Angew. Math. 375-376 (1987), 259-306.

[Mi] J. Milne, Étale Cohomology, Princeton Math. Ser., vol. 33, Princeton University Press, 1980.

[Mo] J. Morales, Trace forms and Stickelberger relations, J. Number Theory 51 (1995), 118-129.

[N1] S. Nakajima, On Galois module structure of the cohomology groups of an algebraic variety, Invent. Math. 75 (1984), 1-8.

[N2] Action of an automorphism of order $p$ on cohomology groups of an algebraic curve, J. Pure Appl. Algebra 48 (1986), 85-94.

[N3] Galois module structure of cohomology groups for tamely ramified coverings of algebraic varieties, J. Number Theory 22 (1986), 115-123.

[S1] J.-P. Serre, Sur la topologie des varietes algebraiques en caracteristique $p$, Symposium Internacional de Topologie Algebraica, Universidad Nacional Autónoma de México and UNESCO, Mexico City, 1958, pp. 24-53. 
[S2]

[S3]

[T]

[W]

[We]
Groupes Algébraiques et Corps de Classes, Hermann, Paris, 1959.

Linear Representations of Finite Groups, Springer-Verlag, New York, 1977.

M. J. Taylor, On Fröhlich's conjecture for rings of integers of tame extensions, Invent. Math. 63 (1981), 41-79.

W. C. Waterhouse, Principal homogeneous spaces and group scheme extensions, Trans. Amer. Math. Soc. 153 (1971), 181-189.

A. Weil, Basic Number Theory, Springer-Verlag, New York, 1967. 